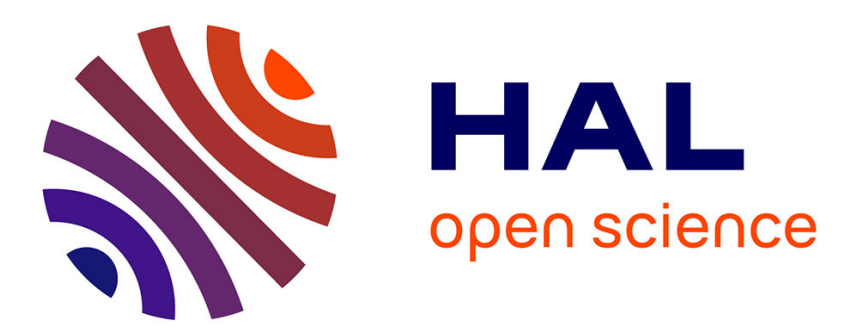

\title{
Comparison of 3-[PP]S Parallel Manipulators based on their Singularity Free Orientation Workspace, Parasitic Motions and Complexity
}

\author{
Abhilash Nayak, Stéphane Caro, Philippe Wenger
}

\section{- To cite this version:}

Abhilash Nayak, Stéphane Caro, Philippe Wenger. Comparison of 3-[PP]S Parallel Manipulators based on their Singularity Free Orientation Workspace, Parasitic Motions and Complexity. Mechanism and Machine Theory, 2018, 129, pp.293-315. 10.1016/j.mechmachtheory.2018.08.001 . hal-01858955

\section{HAL Id: hal-01858955 https://hal.science/hal-01858955}

Submitted on 21 Aug 2018

HAL is a multi-disciplinary open access archive for the deposit and dissemination of scientific research documents, whether they are published or not. The documents may come from teaching and research institutions in France or abroad, or from public or private research centers.
L'archive ouverte pluridisciplinaire $\mathbf{H A L}$, est destinée au dépôt et à la diffusion de documents scientifiques de niveau recherche, publiés ou non, émanant des établissements d'enseignement et de recherche français ou étrangers, des laboratoires publics ou privés. 


\title{
Comparison of 3-[PP]S Parallel Manipulators based on their Singularity Free Orientation Workspace, Parasitic Motions and Complexity
}

\author{
Abhilash Nayak ${ }^{\star}$ \\ abhilash.nayak@ls2n.fr \\ Stéphane Caro $^{\dagger} \quad$ Philippe Wenger $^{\dagger}$ \\ stephane.caro@ls2n.fr philippe.wenger@ls2n.fr \\ $\star$ École Centrale de Nantes \\ $\dagger$ Centre National de la Recherche Scientifique, \\ Laboratoire des Sciences du Numérique de Nantes (LS2N), UMR CNRS 6004, 44321 Nantes, France
}

Keywords: Parallel manipulator, 3-[PP]S, MICR, parasitic motions, Pareto optimal solutions, complexity

\begin{abstract}
The choice of a parallel manipulator for a particular application is a challenging task due to the existence of numerous designs that generate the same motion type. Hence, this paper attempts to compare a class of 3 degreeof-freedom parallel manipulators belonging to the 3-[PP]S family, for which each leg is confined to move in a plane with the first two joints generating a motion equivalent to two coplanar translations. Specifically, the 3-[PP]S parallel manipulators in which the planes are arranged in a Y-pattern are considered and are compared with respect to their singularity-free orientation workspace, parasitic motions within that workspace and complexity indices. Moreover, the comparison is done between the 3-[PP]S-Y family of manipulators for their different operation modes, with different actuation schemes and for different sets of design parameters. Two indices are used to quantify the singularityfree workspace and the parasitic motions of the manipulators at hand. Additionally, the non-dominated manipulator architectures with regard to their singularity-free regions and parasitic motions are highlighted thanks to a Pareto front. Finally, the Pareto-optimal solutions are ranked in ascending order of complexity.
\end{abstract}

\section{Introduction}

In recent years, Parallel Manipulators (PM) have been used in several industrial applications due to their high stiffness and high payload capacity [1, 2, 3, 4]. The six degree-of-freedom (dof) Gough-Stewart platform is one example with many applications like flight simulation, rehabilitation and machining. Besides, lower mobility PMs, i.e., PMs with less than six dof, have gained a lot of attention as six actuators are not required for many applications [5]. Amongst them, the 3-dof mechanisms have been the focus of research for many interesting applications. These manipulators can be classified into different families based on their mobile-platform motion type. DELTA robot [6] and 3-UPU mechanism [7] are some examples of 3-dof translational PMs. The Agile eye is a well known 3-dof spherical PM [8]. PMs with coupled motions generate both translational and rotational motions. An interesting family of 3-dof mechanisms with coupled motions is that composed of zero torsion mechanisms [9, 10, 11]. Human eye, sterno-clavicular joint and human wrist can be modelled as 3-dof zero-torsion parallel mechanisms [9]. The 3RPS PM, introduced by Hunt [2] is one example of zero-torsion mechanism that has been studied extensively. Other examples include the 3-PRS, 3-RRS and 3-PPS PMs. We can find 3-RPS mechanisms as a part of a 6-dof robot [12], as a micro-manipulator wrist subsystem of the ARTISAN manipulator [13] and as a reaction compensation device for space robots [14]. The 3-PRS PM was first proposed for telescopic applications [15]. Another well-known application of the 3-PRS mechanism is a machining head, called the Sprint Z3, developed and patented by DS Technology [16]. The 3-RPS PM was used as a boat simulator with different actuation modes [17]. The 3-RRS PM was analyzed in [18] to be used as a part of a 6-dof hybrid robot for pick-and-place operations. A flexure based 3-PPS PM was used for a nano imprinting tool module in [19]. 
The terminology Zero torsion mechanisms arises from the fact that the rotation of the mobile platform about an axis normal to the latter is always constrained and they display a symmetric motion with respect to the constrained axis. Accordingly, the local motions of the mobile platform of such mechanisms are two non-pure rotations about horizontal axes and sometimes a pure translational motion along a direction normal to the axes of the rotational motions. A popular category of these mechanisms is the 3-[PP]S type, for which each leg is confined to move in a plane with the first two joints generating a motion equivalent to two coplanar translations. When these planes are arranged such that they intersect at $120^{\circ}$ in a common line, they are henceforth addressed as the 3-[PP]S-Y family. If the arrangement of those planes is based on equilateral pattern, they will be part of the so-called 3-[PP]S- $\Delta$ family. If these planes are arranged orthogonally, they are already known as the 3-[PP]S-cube family. For instance, the 3RPS-cube manipulator analyzed in [20] is part of the 3-[PP]S-cube family. Since the number of degree-of-freedom and motion types are the same, the 3-[PP]S PMs can be used for similar applications although their parasitic motions (horizontal displacements due to horizontal axes rotations) differ as it is shown in this paper. This paper aims at finding a common ground to compare the mechanisms belonging to the 3-[PP]S-Y family. Several techniques have already been introduced in the literature to compare PMs. A comparison study of four 3-dof translational PMs was performed by Tsai and Joshi based on their well conditioned workspace, stiffness and inertia properties [21]. Pond and Carretero compared the 3-PRS, 3-RPS and the Tricept PMs by formulating their dimensionally homogeneous square Jacobian matrices and then calculating the condition number and singular values of the Jacobian [22]. Li et al. compared 3-PRS PMs with different limb arrangements with respect to their parasitic motions [23] and listed 1T2R PMs without parasitic motions [24]. In this paper, the comparison of mechanisms belonging to the 3-[PP]S-Y family is performed with respect to their singularity free orientation workspace and their parasitic motions. An index, named Maximum Inscribed Circle Radius (MICR), introduced in [25] is used to quantify the singularity free orientation workspace of the manipulators. The maximum parasitic motion, denoted as $\mu$ within the Maximum Inscribed Circle is used as a second performance index. Furthermore, the manipulators are compared based on their design parameters and actuation scheme. The kinematic and singularity analysis problems are tackled locally using screw theory and globally using algebraic geometry tools [26]. Finally, the Pareto optimal solutions obtained with respect to the two objective functions MICR and $\mu$ are ranked based on their kinematic complexities using the complexity indices defined in $[27,28]$. It should be noted that joint limits and internal collisons are not considered in this paper.

The paper is organized as follows. First, the manipulator architectures under study are described. Section 3 presents the constraint equations for the manipulators belonging to the 3-[PP]S-Y family. Sections 4 deals with the operation mode analysis of the foregoing manipulators. Section 5 presents the kinematic singularities of the manipulators at hand obtained with screw theory approaches. Some illustrative examples are given in Section 6. Finally, the manipulators belonging to the 3-[PP]S-Y family are compared based on the two performance indices introduced in this paper and the obtained Pareto-optimal solutions are highlighted and are ranked in ascending order of complexity.

\section{Manipulators under study}

Figure 1 represents six manipulators from the 3-[PP]S-Y family. The two coplanar translational motions can be obtained with the following serial kinematic chains: RP, PhR, PvR, RR, PvPh or PhPv with Ph denoting a prismatic joint of horizontal direction and $\mathrm{Pv}$ denoting a prismatic joint of vertical direction ${ }^{1}$. Though there are other possible orientations of the prismatic joints [29], most practical applications include horizontal or vertical prismatic joints. The spherical joint centers are restricted to move along the vertical planes $\Pi_{1}, \Pi_{2}$ and $\Pi_{3}$ shown in Fig. 2. These planes are considered in the fixed coordinate frame such that they pass through points $A_{1}, A_{2}$ and $A_{3}$, respectively. They have a common line of intersection, $\mathscr{L}$ along the $z_{0}$-axis and they subtend an angle of $120^{\circ}$. This geometric condition is used to derive the constraint equations for these manipulators. Table 1 gives a list of twelve manipulators belonging to the 3-[PP]S-Y family. The underlined letters denote actuated joints.

Both the base and platform are equilateral triangles with vertices $A_{i}$ and $B_{i}, i=1,2,3$ and with circumradii $h_{1}$ and $h_{2}$, respectively. The origin $O_{0}$ of the fixed coordinate frame $\Sigma_{0}$ coincides with base circumcenter while origin $O_{1}$ of frame $\Sigma_{1}$ coincides with the platform circumcenter. The $x_{0}$-axis of $\Sigma_{0}$ is along line $\left(O_{0} A_{1}\right)$ and the $x_{1}$-axis of frame $\Sigma_{1}$

${ }^{1}$ It should be noted that these directions are considered following the notation defined in [11] and it is assumed that the fixed base is horizontal. 


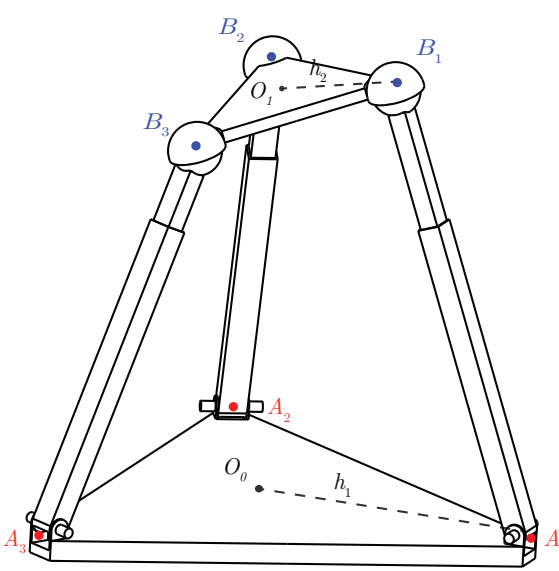

(a) 3-RPS PM

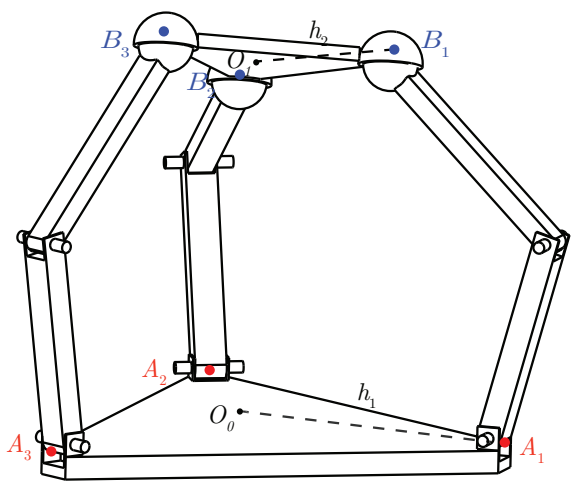

(c) 3-RRS PM

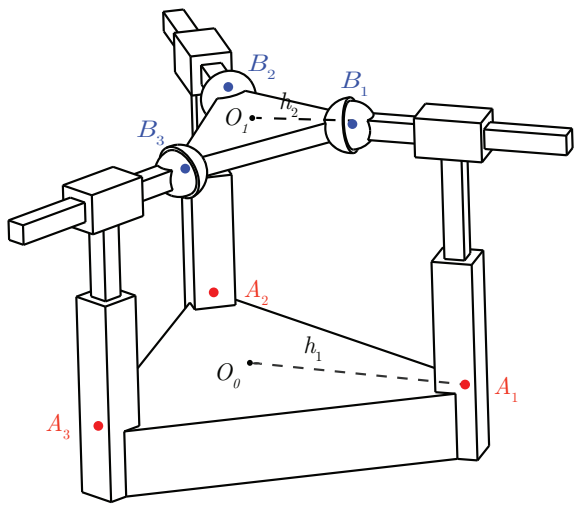

(e) 3-PvPhS PM

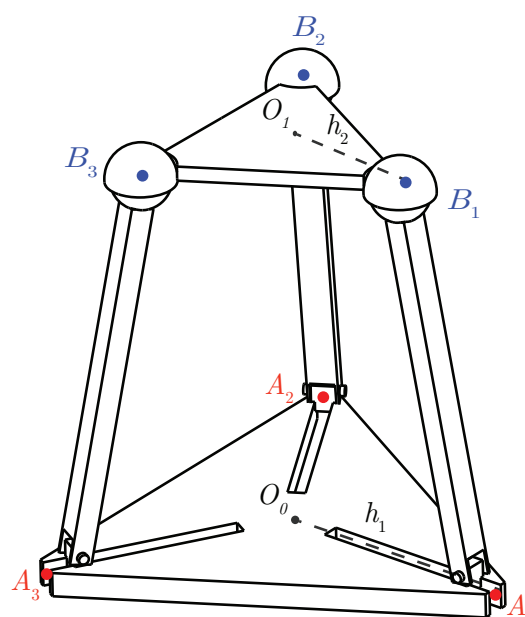

(b) 3-PhRS PM

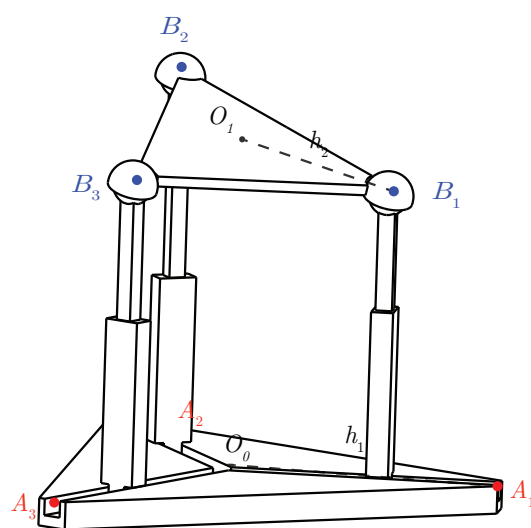

(d) 3-PhPvS PM

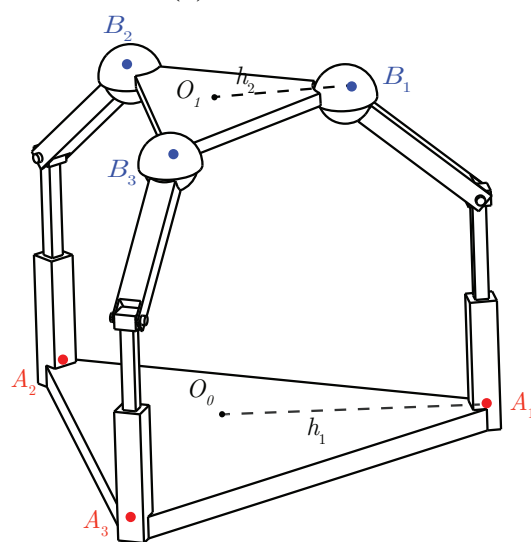

(f) 3-PvRS PM

Figure 1: Six parallel manipulators belonging to the 3-[PP]S-Y family 


\begin{tabular}{|c|c|}
\hline No. & 3-[PP]S-Y family \\
\hline 1 & 3-PvRS \\
\hline 2 & $3-\overline{\mathrm{Ph}} \mathrm{RS}$ \\
\hline 3 & $3-\overline{P v} \underline{R} S$ \\
\hline 4 & 3-Ph్ㅡ $\mathrm{S}$ \\
\hline 5 & 3-RP्PS \\
\hline 6 & $3-\underline{R} \bar{P} S$ \\
\hline 7 & 3- $-\bar{R} \underline{R}$ \\
\hline 8 & $3-\underline{R} \bar{R} S$ \\
\hline 9 & 3-PhPvS \\
\hline 10 & 3-Ph $\overline{\mathrm{Pv} S}$ \\
\hline 11 & 3-PvhS \\
\hline 12 & $3-\mathrm{Pv} \overline{\mathrm{Ph} S}$ \\
\hline
\end{tabular}

Table 1: Manipulators belonging to the 3-[PP]S-Y family

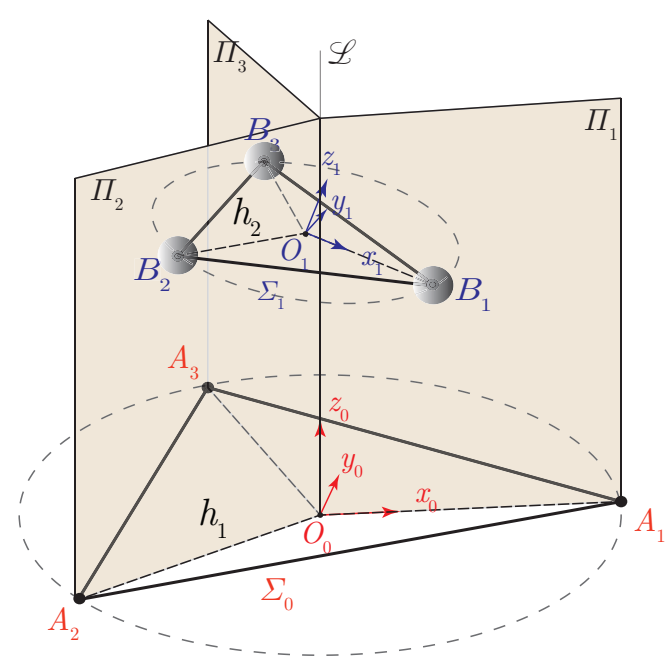

Figure 2: Planes containing the limbs of the $3-[\mathrm{PP}] \mathrm{S}-\mathrm{Y}$ PMs

is along line $\left(O_{1} B_{1}\right)$. Thus, the axes $y_{i}, i=1,2$ are parallel to lines $\left(A_{2} A_{3}\right)$ and $\left(B_{2} B_{3}\right)$, respectively and the axes $z_{i}$, $i=1,2$ are normal to the base and the moving-platform, respectively.

\section{Constraint Equations}

This section aims to determine the constraint equations of the manipulators under study. Those constraint equations are expressed algebraically based on the motions of the moving-platform that are constrained by the legs.

From Fig. 2, the coordinates of points $A_{i}$ and $B_{i}$ in coordinate frames $\Sigma_{0}$ and $\Sigma_{1}$, respectively, are expressed as follows:

$$
\begin{aligned}
& { }^{0} \mathbf{a}_{1}=\left[h_{1}, 0,0\right]^{T}, \quad{ }^{0} \mathbf{a}_{2}=\left[-\frac{1}{2} h_{1}, \frac{\sqrt{3}}{2} h_{1}, 0\right]^{T}, \quad{ }^{0} \mathbf{a}_{3}=\left[-\frac{1}{2} h_{1},-\frac{\sqrt{3}}{2} h_{1}, 0\right]^{T} \\
& { }^{1} \mathbf{b}_{1}=\left[h_{2}, 0,0\right]^{T}, \quad{ }^{1} \mathbf{b}_{2}=\left[-\frac{1}{2} h_{2}, \frac{\sqrt{3}}{2} h_{2}, 0\right]^{T}, \quad{ }^{1} \mathbf{b}_{3}=\left[-\frac{1}{2} h_{2},-\frac{\sqrt{3}}{2} h_{2}, 0\right]^{T}
\end{aligned}
$$

The rotation matrix ${ }^{0} \mathbf{R}_{1}$ from frame $\Sigma_{0}$ to frame $\Sigma_{1}$ is expressed as:

$$
{ }^{0} \mathbf{R}_{1}=\left[\begin{array}{ccc}
x_{0}{ }^{2}+x_{1}{ }^{2}-x_{2}{ }^{2}-x_{3}{ }^{2} & -2 x_{0} x_{3}+2 x_{1} x_{2} & 2 x_{0} x_{2}+2 x_{1} x_{3} \\
2 x_{0} x_{3}+2 x_{1} x_{2} & x_{0}^{2}-x_{1}{ }^{2}+x_{2}{ }^{2}-x_{3}{ }^{2} & -2 x_{0} x_{1}+2 x_{3} x_{2} \\
-2 x_{0} x_{2}+2 x_{1} x_{3} & 2 x_{0} x_{1}+2 x_{3} x_{2} & x_{0}^{2}-x_{1}{ }^{2}-x_{2}{ }^{2}+x_{3}{ }^{2}
\end{array}\right]
$$

where $x_{j}, j=0,1,2,3$ are the unit orientation quaternions satisfying: $x_{0}^{2}+x_{1}^{2}+x_{2}^{2}+x_{3}^{2}=1$.

From Fig. 2, for each leg, the vector connecting points $A_{i}$ and $B_{i}$ should always lie in the plane $\Pi_{i}$. This geometrical constraint holds true for all manipulators belonging to the 3-[PP]S-Y family. To derive the constraint equations, we can express the normal vector $\mathbf{s}_{i}$ to planes $\Pi_{i}, i=1,2,3$, in frame $\Sigma_{0}$ as follows:

$$
{ }^{0} \mathbf{s}_{1}=[0,-1,0]^{T}, \quad{ }^{0} \mathbf{s}_{2}=\left[\frac{\sqrt{3}}{2}, \frac{1}{2}, 0\right]^{T}, \quad{ }^{0} \mathbf{s}_{3}=\left[-\frac{\sqrt{3}}{2}, \frac{1}{2}, 0\right]^{T}
$$

In the fixed coordinate frame $\Sigma_{0}$, the coordinates of point $B_{i}$ can be expressed as follows:

$$
{ }^{0} \mathbf{b}_{i}={ }^{0} \mathbf{R}_{1}{ }^{1} \mathbf{b}_{i}+{ }^{0} \mathbf{p}_{1}, \quad i=1,2,3
$$


where ${ }^{0} \mathbf{p}_{1}$ is the point-displacement vector from the origin $O_{0}$ of $\Sigma_{0}$ to the origin $O_{1}$ of $\Sigma_{1}$ expressed in $\Sigma_{0}$ as $[X, Y, Z]^{T}$. The vector ${\overrightarrow{A_{i} B_{i}}}_{i}$ is perpendicular to $\mathbf{s}_{i}$, which is expressed as $\left({ }^{0} \mathbf{b}_{i}-{ }^{0} \mathbf{a}_{i}\right)^{T}{ }^{0} \mathbf{s}_{i}=0$ which after simplification yields the following three equations:

$$
\begin{aligned}
& g_{1}:=x_{0} x_{3}=0 \\
& g_{2}:=X-h_{2} x_{1}^{2}+h_{2} x_{2}^{2}=0 \\
& g_{3}:=Y-4 h_{2} x_{0} x_{3}+2 h_{2} x_{1} x_{2}=0
\end{aligned}
$$

Depending upon the actuated joints, three other equations, $g_{4}=0, g_{5}=0$ and $g_{6}=0$ can be derived. For instance, in case of the 3-RPS PM (Figure 1a), the Euclidean distance between $A_{i}$ and $B_{i}$ must be equal to the prismatic joint length, $r_{i}$ for the $i$-th leg of the manipulator. As a result, $\left\|{ }^{0} \mathbf{b}_{i}-{ }^{0} \mathbf{a}_{i}\right\|^{2}=r_{i}^{2}$ leads to three additional equations [26] :

$$
\begin{aligned}
g_{4}:= & \left(4 X h_{2}-4 h_{1} h_{2}\right) x_{0}^{2}-4 Z h_{2} x_{0} x_{2}+4 Y h_{2} x_{0} x_{3}+\left(4 X h_{2}-4 h_{1} h_{2}\right) x_{1}^{2}+4 Y h_{2} x_{1} x_{2}+4 Z h_{2} x_{1} x_{3} \\
& +X^{2}-2 X h_{1}-2 X h_{2}+Y^{2}+Z^{2}+h_{1}^{2}+2 h_{1} h_{2}+h_{2}^{2}-r_{1}^{2}=0 \\
g_{5}:= & \left(-2 \sqrt{3} Y h_{2}-2 X h_{2}-4 h_{1} h_{2}\right) x_{0}^{2}-2 \sqrt{3} Z h_{2} x_{0} x_{1}+2 Z h_{2} x_{0} x_{2}+\left(2 \sqrt{3} X h_{2}-2 Y h_{2}\right) x_{0} x_{3} \\
& +\left(-2 X h_{2}-h_{1} h_{2}\right) x_{1}^{2}+\left(-2 \sqrt{3} X h_{2}-2 \sqrt{3} h_{1} h_{2}-2 Y h_{2}\right) x_{1} x_{2}-2 Z h_{2} x_{1} x_{3}+\left(-2 \sqrt{3} Y h_{2}-3 h_{1} h_{2}\right) x_{2}^{2} \\
& -2 \sqrt{3} Z h_{2} x_{2} x_{3}+\sqrt{3} Y h_{1}+\sqrt{3} Y h_{2}+X^{2}+X h_{1}+X h_{2}+Y^{2}+Z^{2}+h_{1}^{2}+2 h_{1} h_{2}+h_{2}^{2}-r_{2}^{2}=0 \\
g_{6}:= & \left(2 \sqrt{3} Y h_{2}-2 X h_{2}-4 h_{1} h_{2}\right) x_{0}^{2}+2 \sqrt{3} Z h_{2} x_{0} x_{1}+2 Z h_{2} x_{0} x_{2}+\left(-2 \sqrt{3} X h_{2}-2 Y h_{2}\right) x_{0} x_{3} \\
& +\left(-2 X h_{2}-h_{1} h_{2}\right) x_{1}^{2}+\left(2 \sqrt{3} X h_{2}+2 \sqrt{3} h_{1} h_{2}-2 Y h_{2}\right) x_{1} x_{2}-2 Z h_{2} x_{1} x_{3}+\left(2 \sqrt{3} Y h_{2}-3 h_{1} h_{2}\right) x_{2}^{2} \\
& +2 \sqrt{3} Z h_{2} x_{2} x_{3}-\sqrt{3} Y h_{1}-\sqrt{3} Y h_{2}+X^{2}+X h_{1}+X h_{2}+Y^{2}+Z^{2}+h_{1}^{2}+2 h_{1} h_{2}+h_{2}^{2}-r_{3}^{2}=0
\end{aligned}
$$

In addition, the normalization equation of the unit quaternion is to be considered, namely,

$$
g_{7}:=x_{0}{ }^{2}+x_{1}{ }^{2}+x_{2}{ }^{2}+x_{3}{ }^{2}-1=0
$$

It is noteworthy that Eq. (5) reveals that the 3-[PP]S-Y family of PMs can have at least two operation modes characterized by $x_{0}=0$ and $x_{3}=0$. The following section examines the primary decomposition of the ideal of constraint equations to identify different operation modes of the mechanisms under study.

\section{Operation Modes}

The constraint equations (5) to (7) describe the configuration space of the manipulators. The first constraint equation is recalled:

$$
x_{0} x_{3}=0
$$

As a result, their configuration space can be split into two parts $x_{0}=0$ and $x_{3}=0$, called the operation modes [29, $30,31]$ separated by a constraint or C-space singularity [32]. To obtain other equations in each operation mode, a polynomial ideal is defined consisting of equations $g_{1}, g_{2}, g_{3}$ and $g_{7}$ with variables $\left\{x_{0}, x_{1}, x_{2}, x_{3}, X, Y, Z\right\}$ over the coefficient ring $\mathbb{C}\left[h_{1}, h_{2}\right]$ as follows:

$$
\mathscr{J}=\left\langle g_{1}, g_{2}, g_{3}, g_{7}\right\rangle
$$

The primary decomposition of ideal $\mathscr{J}$ results in two ideals $\mathscr{J}_{k}(i=1,2)$ confirming two operation modes for all the PMs in the 3-[PP]S-Y family, no matter their actuation scheme.

The analysis is completed by adding the remaining constraint equations $g_{4}=g_{5}=g_{6}=0$ to the primary ideals $\mathscr{J}_{1}$ and $\mathscr{J}_{2}$, which returns two ideals $\mathscr{K}_{1}$ and $\mathscr{K}_{2}$. As a consequence, the ideals $\mathscr{K}_{i}$ correspond to the two operation modes and can be studied separately.

$$
\mathscr{K}_{k}=\mathscr{J}_{k} \cup\left\langle g_{4}, g_{5}, g_{6}\right\rangle \quad k=1,2
$$


Ideal $\mathscr{K}_{1}$-Operation mode 1 , OM1 $\left(x_{0}=0\right)$ : The moving platform is always found to be displaced about a finite screw axis by 180 degrees from the identity position where $\Sigma_{0}$ coincides with $\Sigma_{1}$ [30]. Substituting $x_{0}=0$ and solving for $X, Y$ from the ideal $\mathscr{K}_{1}$ shows that the translational motions can be parametrized by $Z$ and the rotational motions by $x_{1}, x_{2}$ and $x_{3}$ along with $x_{1}^{2}+x_{2}^{2}+x_{3}^{2}=1[30]$.

Ideal $\mathscr{K}_{2}$-Operation mode 2, OM2 $\left(x_{3}=0\right)$ : The moving platform is displaced about a finite screw axis with a rotation angle $\alpha$ defined as: $\alpha= \pm 2 \operatorname{acos}\left(x_{0}\right)$. The screw axis in this case stays parallel to the xy-plane [30]. Substituting $x_{3}=0$ and solving for $X, Y, Z$ from the ideal $\mathscr{K}_{2}$ shows that the translational motions can be parametrized by $Z$ and the rotational motions by $x_{0}, x_{1}$ and $x_{2}$ along with $x_{0}^{2}+x_{1}^{2}+x_{2}^{2}=1$ [30].

Therefore, PMs belonging to the 3-[PP]S-Y family exhibit two operation modes. In what remains, those manipulators are compared based on their singularity-free orientation workspace size and parasitic motions.

\section{Singularity Analysis and Parasitic Motions}

For each operation mode, the motion of the moving platform can be parametrized by $Z$ and any two of the orientation parameters $x_{i}$ [29]. It simplifies the singularity analysis and the singular surfaces can be visualized in the three dimensional Euclidean space, $\mathbb{E}^{3}$. However, the rotations of the moving-platform are not pure. Indeed some undesired translational motions called as parasitic motions, arise. This section is dedicated to the evaluation of the singularity-free orientation workspace and the parasitic motions of PMs belonging to the 3-[PP]S-Y family. The forward kinematic Jacobian matrix is derived in two ways: $(i)$ differentiating the constraint equations with respect to the output variables (ii) using screw theory. It is shown how screw theory methods simplify the determination of the Jacobian matrix for different actuation schemes. An index is defined to assess the maximum singularity-free orientation workspace of the mechanisms under study. Additionally, their parasitic motions are also assessed.

\subsection{Jacobian Matrix obtained by differentiating the constraint equations}

The Jacobian matrix is defined for each operation mode as a matrix composed of the first order partial derivatives of the constraint equations with respect to the parameters $x_{0}, x_{1}, x_{2}, x_{3}, X, Y$ and $Z[33,34]$, i.e.,

$$
\mathbf{J}_{k}=\left(\frac{\partial g_{m}}{\partial x_{i}}, \frac{\partial g_{m}}{\partial X}, \frac{\partial g_{m}}{\partial Y}, \frac{\partial g_{m}}{\partial Z}\right) \text { where } k=1,2 ; \quad m=1, \ldots, 7 ; \quad i=0, \ldots, 3
$$

where, $g_{m}, m=1, \ldots, 7$ are the constraint equations for the manipulator at hand. Thus, the manipulator reaches a kinematic singularity when $\operatorname{det}\left(\mathbf{J}_{k}\right)=0$. The vanishing condition of $\operatorname{det}\left(\mathbf{J}_{k}\right)$ results in a hyper-variety for each operation mode:

$$
S_{1}: x_{3} \cdot f_{1}\left(x_{1}, x_{2}, x_{3}, X, Y, Z\right)=0 \quad \text { and } \quad S_{2}: x_{0} \cdot f_{2}\left(x_{0}, x_{1}, x_{2}, X, Y, Z\right)=0
$$

The polynomials $f_{1}$ and $f_{2}$, whose degree depends on the PM at hand describe the singularities in each operation mode, which depend on the actuation scheme. $x_{0}=x_{3}=0$ describes the constraint singularity that exhibits the transition between $\mathscr{K}_{1}$ and $\mathscr{K}_{2}$.

It is noteworthy that this method is not always convenient to use as equations $g_{4}, g_{5}$ and $g_{6}$ depend on the manipulator actuation scheme. For instance, the equations for the 3-RPS manipulator expressed in Sec. 3 depend on the actuated prismatic joint lengths. Besides, it should be noted that the derivation of equations $g_{4}, g_{5}$ and $g_{6}$ as a function of the actuated revolute joint angles is usually tedious. To overcome this problem, screw theory techniques are adopted to derive the Jacobian matrices of the PMs.

\subsection{Jacobian Matrix obtained based on Screw Theory}

Based on the theory of reciprocal screws, the velocity model of a parallel manipulator can be expressed as

$$
\mathbf{A t}=\mathbf{B} \dot{\rho}
$$

where $\dot{\rho}$ is the actuated joint rate vector. $\mathbf{t}$ is the moving platform twist with respect to the fixed base i.e., it contains the angular velocity vector of the moving platform and the linear velocity vector of the MP geometric center. $\mathbf{A}$ is called the forward Jacobian matrix and it incorporates the actuation and constraint wrenches of the PM while B is 
named the inverse Jacobian matrix [35]. For a non redundantly actuated and non overconstraint PM, A is a $6 \times 6$ square matrix whereas $\mathbf{B}$ is a $6 \times n$ matrix where $n$ is the number of actuated joints. To compare the manipulators of the 3-[PP]S-Y family, only the parallel singularities are considered such that, $\mathbf{A}$ is rank deficient. Since, $\mathbf{A}$ is square, configurations satisfying $\operatorname{det}(\mathbf{A})=0$ are singular. Moreover, the manipulators belonging to the 3-[PP]S family cannot meey any serial singularity corresponding to the rank deficiency of $\mathbf{B}$ except the 3-RRS PM.

For instance, the kinematic modeling of the 3-Rㅛ and 3-RPS PMs following Eq. 18 can be expressed as

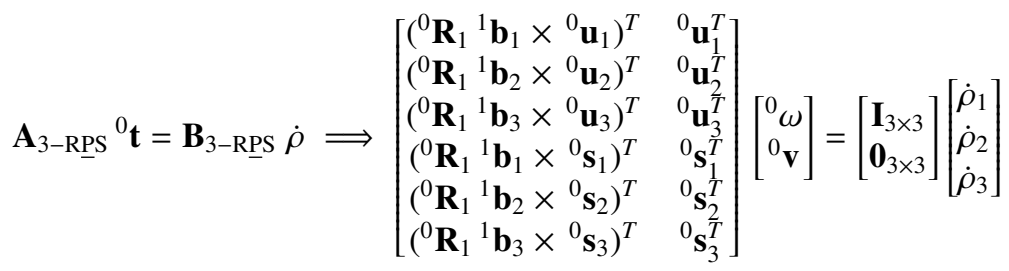

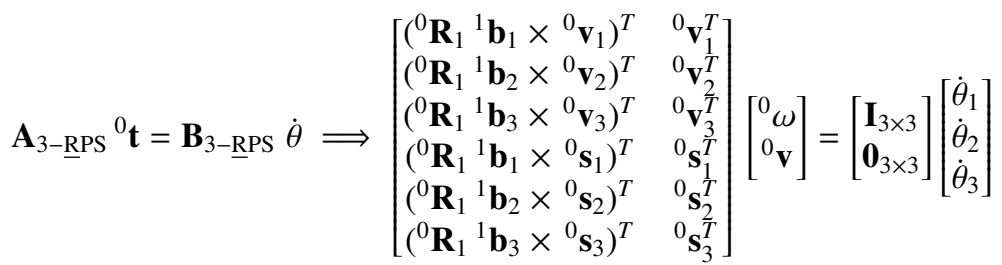

where, ${ }^{0} \mathbf{u}_{i}$ is a unit vector along the prismatic joint direction and expressed in $\Sigma_{0},{ }^{0} \mathbf{s}_{i}$ is the unit vector along the $i$ th revolute joint axis, ${ }^{0} \mathbf{v}_{i}$ is a vector normal to both ${ }^{0} \mathbf{u}_{i}$ and ${ }^{0} \mathbf{s}_{i}$. Matrices $\mathbf{A}_{3 \text {-RPS }}$ and $\mathbf{A}_{3-\text { RPS }}$ incorporate the actuation and constraint wrenches of the 3-RPS and 3-RPS PMs, respectively [35] and they are both $6 \times 6$ square matrices. For the $i$-th leg of these manipulators, the constraint wrench, ${ }^{0} \hat{\zeta}_{0 i}^{c}$ and the actuation wrench, ${ }^{0} \hat{\zeta}_{0 i}^{a}$ are represented in Figs. 3 and 4 and are expressed in frame $\Sigma_{0}$ as follows:

$$
\begin{aligned}
& 3 \text { - RPS PM : }{ }^{0} \hat{\zeta}_{0 i}^{c}=\left[\begin{array}{c}
{ }^{0} \mathbf{s}_{i} \\
{ }^{0} \mathbf{R}_{1}{ }^{1} \mathbf{b}_{i} \times{ }^{0} \mathbf{s}_{i}
\end{array}\right] \quad{ }^{0} \hat{\zeta}_{0 i}^{a}=\left[\begin{array}{c}
{ }^{0} \mathbf{u}_{i} \\
{ }^{0} \mathbf{R}_{1}{ }^{1} \mathbf{b}_{i} \times{ }^{0} \mathbf{u}_{i}
\end{array}\right]
\end{aligned}
$$

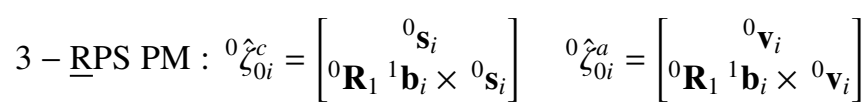

Thus, from Eqs. (1), (3) and (4), the determinant of the forward Jacobian matrix $\mathbf{A}_{3-\mathrm{RPS}}$ and $\mathbf{A}_{3-\mathrm{RPS}}$ in Eqs. (19) and (20) can be calculated in terms of $x_{0}, x_{1}, x_{2}, x_{3}, X, Y, Z$ and the design parameters. The derivation of the forward Jacobian matrix by differentiating the constraint equations is tedious when a revolute joint is actuated whereas using screw theory, it is straightforward regardless of the actuation scheme. Therefore, the forward singularities for different actuation schemes of 3-[PP]S PMs are determined when the determinant of matrix $\mathbf{A}$ in Eq. (18) vanishes.

\subsection{Singularity loci in the orientation workspace}

The singularities can be expressed in the orientation workspace by parametrizing the orientation of the platform in terms of Tilt-and-Torsion (TET) angles, $\phi$ (azimuth), $\theta$ (tilt) and $\sigma$ (torsion) $[9,36]$ as recalled in Fig. $5^{2}$. As shown in [9], those angles are more suitable for 3-[PP]S manipulators, which are known to be zero torsion mechanisms ( $\sigma$ is always equal to 0 or $\pi$ depending on the operation mode). The orientation quaternions can be expressed in terms of

${ }^{2}$ Figure 5 is a combination of Figs. 1(a) and 1(b) in [9]. In case of ZXZ Euler angle notation, a rotation matrix is decomposed as $\mathbf{R}=$ $\mathbf{R}_{z}(\phi) \mathbf{R}_{x}(\theta) \mathbf{R}_{z}(\psi)$ whereas $T \mathcal{E} T$ angles use only two rotations $\mathbf{R}=\mathbf{R}_{a}(\theta) \mathbf{R}_{z}(\sigma)$, where $\theta$ and $\sigma$ represent tilt and torsion, respectively. That is the reason why they are named as Tilt and Torsion angles. However, it should be noted that the azimuth angle is necessary to locate the axis- $a$ and is measured from $x$-axis to be consistent with the ZXZ Euler angle convention. This simple yet effective change of angles makes the whole analysis much simpler and suits the behavior of any zero-torsion mechanism. 


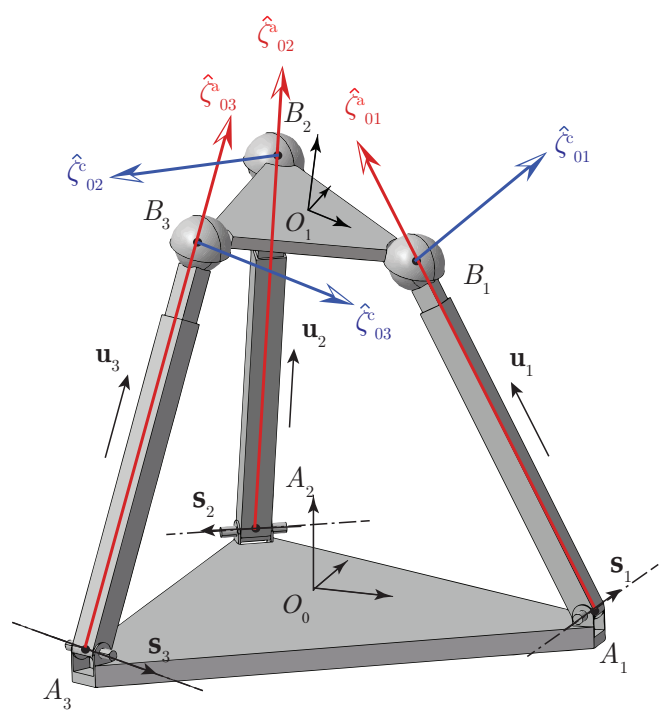

Figure 3: Actuation and constraint wrenches for the 3-RPS PM

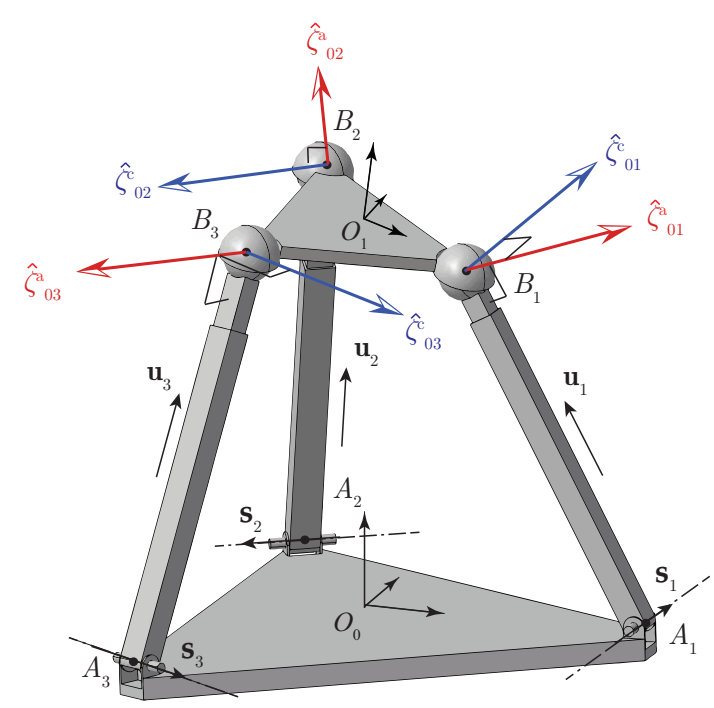

Figure 4: Actuation and constraint wrenches for the 3-RPS PM

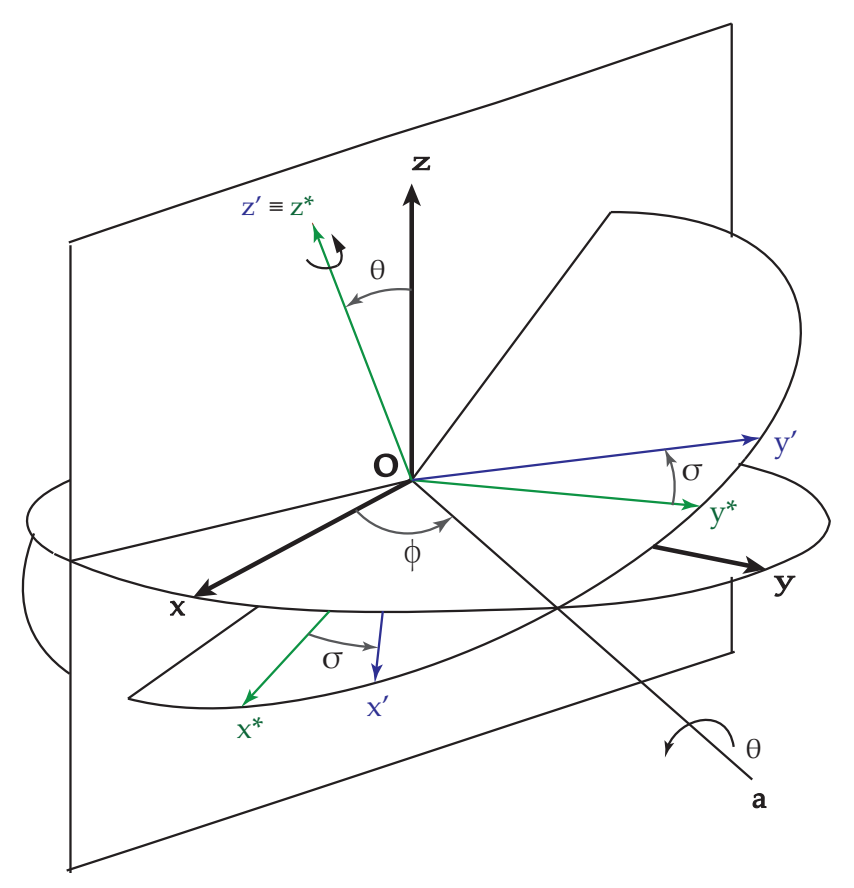

Figure 5: Tilt and Torsion angles [9] 
the $T \mathcal{F} T$ angles as follows ${ }^{3}$ :

$$
\begin{aligned}
& x_{0}=\cos \left(\frac{\theta}{2}\right) \cos \left(\frac{\sigma}{2}\right) \\
& x_{1}=\sin \left(\frac{\theta}{2}\right) \cos \left(\phi-\frac{\sigma}{2}\right) \\
& x_{2}=\sin \left(\frac{\theta}{2}\right) \sin \left(\phi-\frac{\sigma}{2}\right) \\
& x_{3}=\cos \left(\frac{\theta}{2}\right) \sin \left(\frac{\sigma}{2}\right)
\end{aligned}
$$

The change of parametrization results in only three parameters expressing the orientation of the moving-platform. Furthermore, for operation mode $\mathscr{K}_{1}$, characterized by $x_{0}=0$, Eq. (23) gives $\sigma=\pi$. Similarly, for operation mode $\mathscr{K}_{2}$, characterized by $x_{3}=0$, Eq. (23) gives $\sigma=0$. Substituting the values of $\sigma$ in the remaining equations, leads to $x_{i}$ expressed as functions of $\phi$ and $\theta$ angles, only.

In order to plot the singularity loci of the manipulator into the orientation workspace, the determinant of the forward Jacobian matrix of the manipulator should be expressed as a function $\phi$ and $\theta$ angles, only. All remaining variables should be eliminated from the determinant of the forward Jacobian matrix. $X$ and $Y$ can be eliminated using Eqs. (6) and (7) for the 3-[PP]S-Y PMs as follows:

$$
X=h_{2}\left(x_{1}^{2}-x_{2}^{2}\right) \quad Y=-2 h_{2} x_{1} x_{2}
$$

To this end, the determinant of the forward Jacobian matrix is a function of $Z, x_{i}, i=0,1,2,3$ and of the actuated joint variables. The actuated joint variables can be eliminated using equations $g_{4}=g_{5}=g_{6}=0$ as shown in Sec. 3 . Additionally, from Eq. (23), $x_{i}$ can be expressed as functions of $\phi$ and $\theta$ angles leading to the final expression of the determinant of the forward Jacobian matrix in terms of $Z, \theta$ and $\phi$.

\subsection{Maximum Inscribed Circle Radius (MICR)}

From a practical point of view, it is desirable to use a PM in its singularity free region. A performance index, named Maximum Inscribed Circle Radius (MICR) and defined in [25], is used in this section to quantify the capability of the Parallel Manipulator (PM) in terms of orientation motions. For a given altitude $\mathrm{Z}$, this index gives the maximum tilt angle $\theta$ that the moving-platform can reach for any azimuth angle $\phi$ without reaching any parallel singularity:

$$
M I C R=\max _{0 \leq \theta \leq \pi}\{\theta, \forall \phi \in[0, \pi], \operatorname{det}(\mathbf{A}) \neq 0\}
$$

MICR values are calculated for all the mechanisms at hand in their operation modes for three ratios for $\frac{h_{2}}{h_{1}}=\left\{\frac{1}{2}, 1,2\right\}$ and two ratios for $\frac{Z}{h_{1}}=1,2$. The ratios $\frac{Z}{h_{1}}$ and $\frac{h_{2}}{h_{1}}$ make sure that the MICR values can be used to compare the mechanisms and their operation modes no matter the platform and base sizes. Thus, MICR is used to compare the PMS belonging to the 3-[PP]S-Y family.

\subsection{Parasitic Motions}

For the 3-[PP]S-Y family of PMs, the workspace is expressed in terms of $Z, \phi$ and $\theta$. Since the torsion angle $\sigma$ can only attain the values 0 or $\pi$ depending on the operation mode, the constrained motions are along the $x$ and $y$ axes. The parasitic displacements along the $x$ and $y$ coordinates of the moving platform are expressed in terms of $Z$ and $x_{i}$ in Equations (24).

\footnotetext{
${ }^{3}$ The change of parameters from quaternions to TET angles is done for the following reasons: quaternions are used to obtain algebraic constraint equations so that the algebraic geometry techniques can be used to analyse those equations. For instance, primary decomposition of the ideal of the constraint polynomials described in Eq. (14) yields the exact number of operation modes and their characterization. However, the parametrization is changed to TET angles to be able to visualize the singularities and maximum parasitic displacements in the orientation workspace. Moreover, they offer a more intuitive approach to deal with zero-torsion mechanisms that are compared in this paper.
} 
To compare the parasitic motions of PMs belonging to the 3-[PP]S-Y family, the maximum displacement within the Maximum Inscribed Circle (MIC) [25] is considered. Here, the following index is defined to quantify the parasitic motions of the moving-platform along $x_{0}$ and $y_{0}$ axes. A parameter with units of length is defined such that

$$
\mu=\max \left(\sqrt{X^{2}+Y^{2}}\right) \quad \text { when } 0 \leq \theta \leq M I C R ;-\pi \leq \phi \leq \pi
$$

$\mu$ can be expressed in terms of $h_{2}, \theta$ and $\phi$ for these manipulators.

\subsection{Internal collisions}

A drawback of using parallel manipulators for potential applications is the existence of internal collisions. It also applies to the PMs belonging to the 3-[PP]S-Y family listed in Table 1. For these manipulators, three kinds of internal collisions should be considered:

1. Collisions between limbs.

2. Collisions between limbs and the moving or fixed platform triangles.

3. Collision between the fixed and moving-platform triangles.

An accurate collision analysis requires the shape and thickness of the limbs as well as the range of motion of the spherical joints. It should be noted that this paper mainly focuses on the singularity free orientation workspace and the parasitic motions. The collision analysis is left for future work.

However, it can be observed that most applications of the 3-[PP]S-Y PMs [15, 16, 18, 19] require them to be in the second operation mode (characterized in this paper by $x_{3}=0$ ), due to the existence of internal collisions in their first operation mode $\left(x_{0}=0\right)$. This is partially accounted for a new complexity index introduced on the next section.

\section{Example: The 3-RPS PM}

In this section, the 3-RPS PM is considered and the forward Jacobian matrix is formulated, leading to the determination of MICR for different sizes of its fixed base and moving platforms. Furthermore, the parasitic motions are visualized within the Maximum Inscribed Circle (MIC) by plotting $\mu$ vs. $\phi$ and $\theta^{4}$.

The architecture of the 3-RPS PM belonging to the family of 3-[PP]S-Y of PMs is shown in Figure 6.

The determinant of $\mathbf{A}_{3-\text { RPS }}$ calculated from Eq. (19) turns out to be a function of $x_{0}, x_{1}, x_{2}, x_{3}, X, Y, Z, h_{1}$ and $h_{2} . X$ and $Y$ are substituted from Eq. (24) and then TEST angle substitutions are done (Eq. (23)) resulting in the determinant of the forward Jacobian matrix to be a function of $Z, h_{1}, h_{2}, \theta, \phi$ and $\sigma$. For operation mode 1 , substituting $\sigma=\pi$ and assigning $h_{1}=1$ and $h_{2}=2$, the implicit plot of the Jacobian can be drawn by varying $Z, \phi$ and $\theta$ as shown in Fig. 7a. For operation mode 2 with $\sigma=0$, the implicit plot is shown in Fig. 7b. A slice of these surfaces at $Z=h_{1}=1$ shows the singularity loci in Fig. 8.

Along with the singularity loci, the so called intersection loci, depicting the collision between each pair of limbs $A_{1} B_{1}, A_{2} B_{2}$ and $A_{3} B_{3}$ are also plotted. They are calculated as follows:

The limbs are assumed to have no thickness and the spherical joints are assumed to have full range of motion. Thus, the problem is reduced to find all possible intersections between three pairs of line segments. For instance, to find the intersection loci of line segments $A_{1} B_{1}$ and $A_{2} B_{2}$, their vector equations are written as follows:

$$
\begin{array}{ll}
\mathbf{r}_{1}=\mathbf{a}_{1}+\left(\mathbf{b}_{1}-\mathbf{a}_{1}\right) t_{1} & t_{1} \in[0,1] \\
\mathbf{r}_{2}=\mathbf{a}_{2}+\left(\mathbf{b}_{2}-\mathbf{a}_{1}\right) t_{2} & t_{2} \in[0,1]
\end{array}
$$

where $\mathbf{r}_{1}$ and $\mathbf{r}_{1}$ are position vectors of a point on line segments $A_{1} B_{1}$ and $A_{2} B_{2}$, respectively. An intersection occurs when $\mathbf{r}_{1}=\mathbf{r}_{2}$. Eliminating $t_{1}$ and $t_{2}$ from this equation gives an implicit curve in terms of $\theta$ and $\phi$ where the considered line segments intersect under the condition $t_{1}, t_{2} \in[0,1]$. The intersection between the other two pairs of limbs are determined in a similar manner, resulting in the intersection loci as plotted in blue in Fig. 8. It is apparent that the

\footnotetext{
${ }^{4}$ Radian is used as the angular unit throughout the text but degree is used in figures for better clarity.
} 


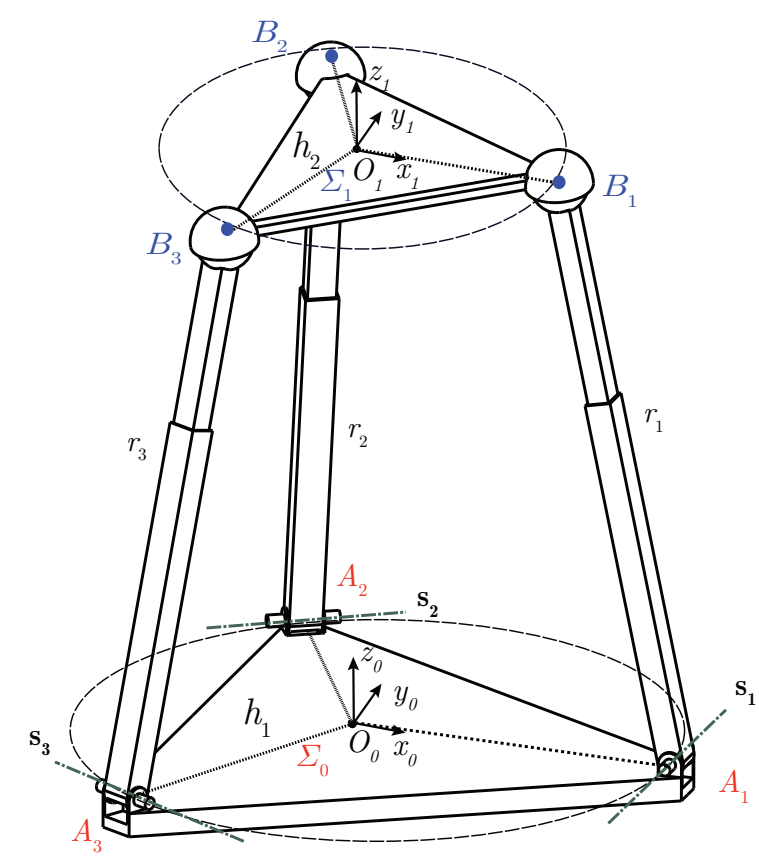

Figure 6: 3-Rㅗ PM architecture

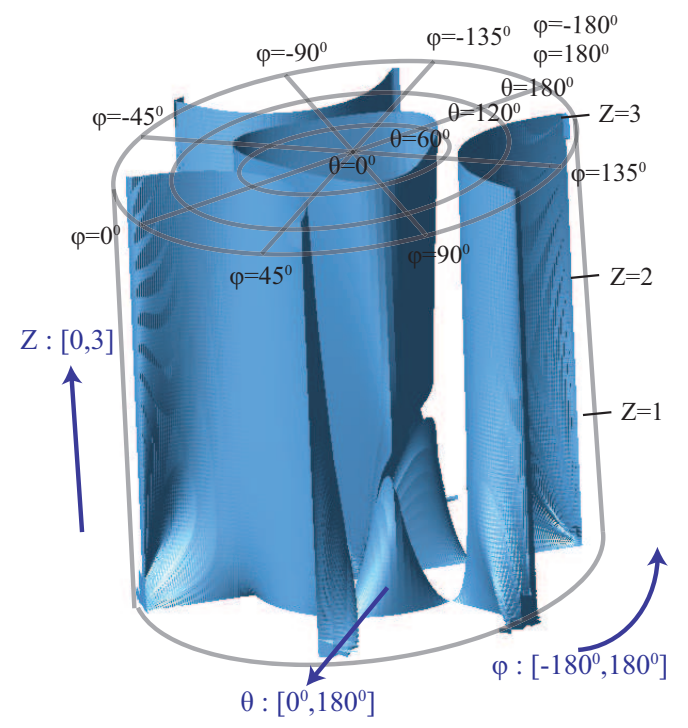

(a) Operation mode 1

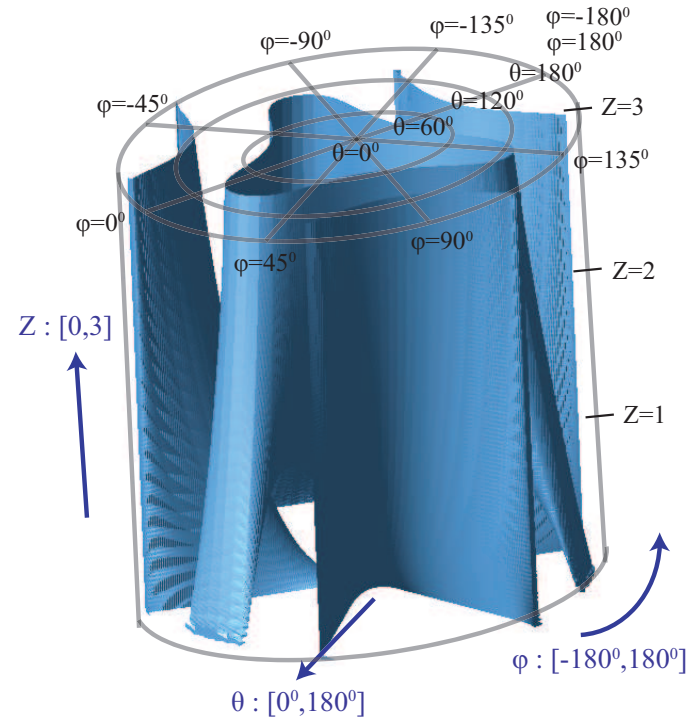

(b) Operation mode 2

Figure 7: 3-RPS singularity surfaces for $\frac{h_{2}}{h_{1}}=2$ 
internal collisions dominate in operation mode 1 leading the a MICR value without practical use. Nonetheless, it is possible to establish a singularity and collision-free workspace by releasing the constraint associated to MIC center lying at $\theta=\phi=0 \mathrm{deg}$. However, this is not the scope of this paper and is left for future work.

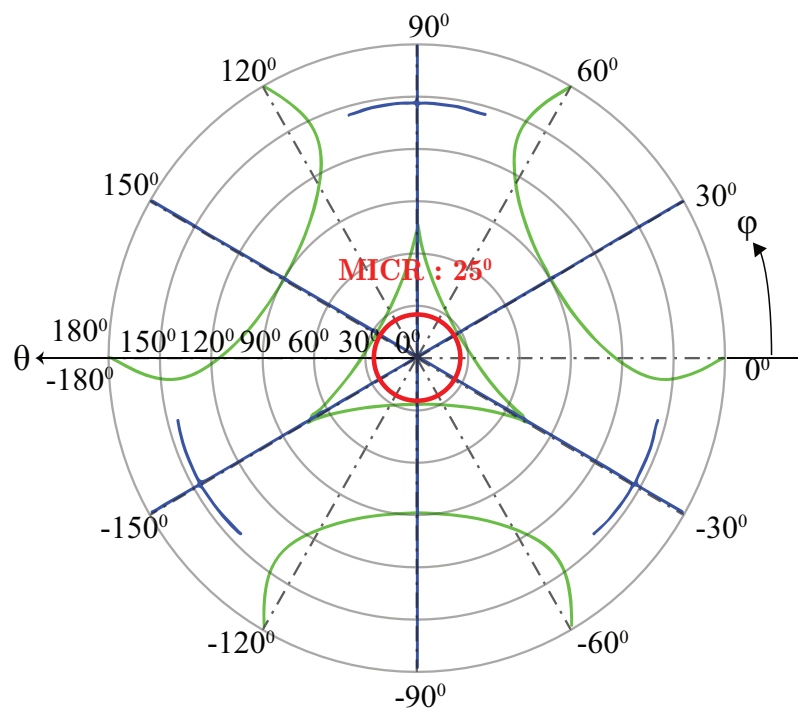

(a) Operation mode 1

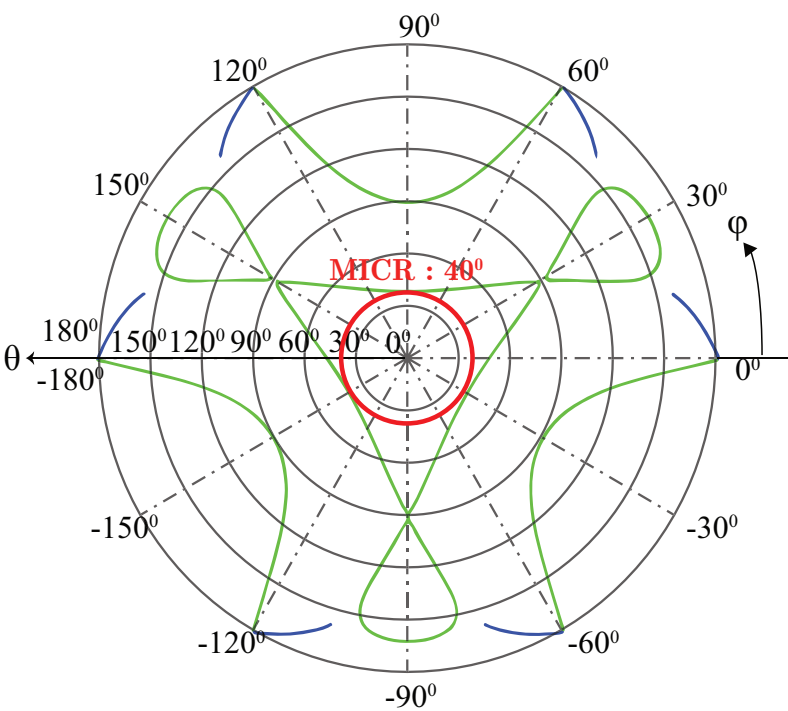

(b) Operation mode 2

Figure 8: 3-RPS singularity loci (green), intersection loci (blue) and the maximum inscribed singularity-free circle $\left(\right.$ red), $\frac{h_{2}}{h_{1}}=2, \frac{Z}{h_{1}}=1$

MICR, defined in Eq. (25). is also marked in these figures. For different ratios of $h_{2}$ to $h_{1}$, the variations in MICR values are plotted in Figs. 9 and 10.

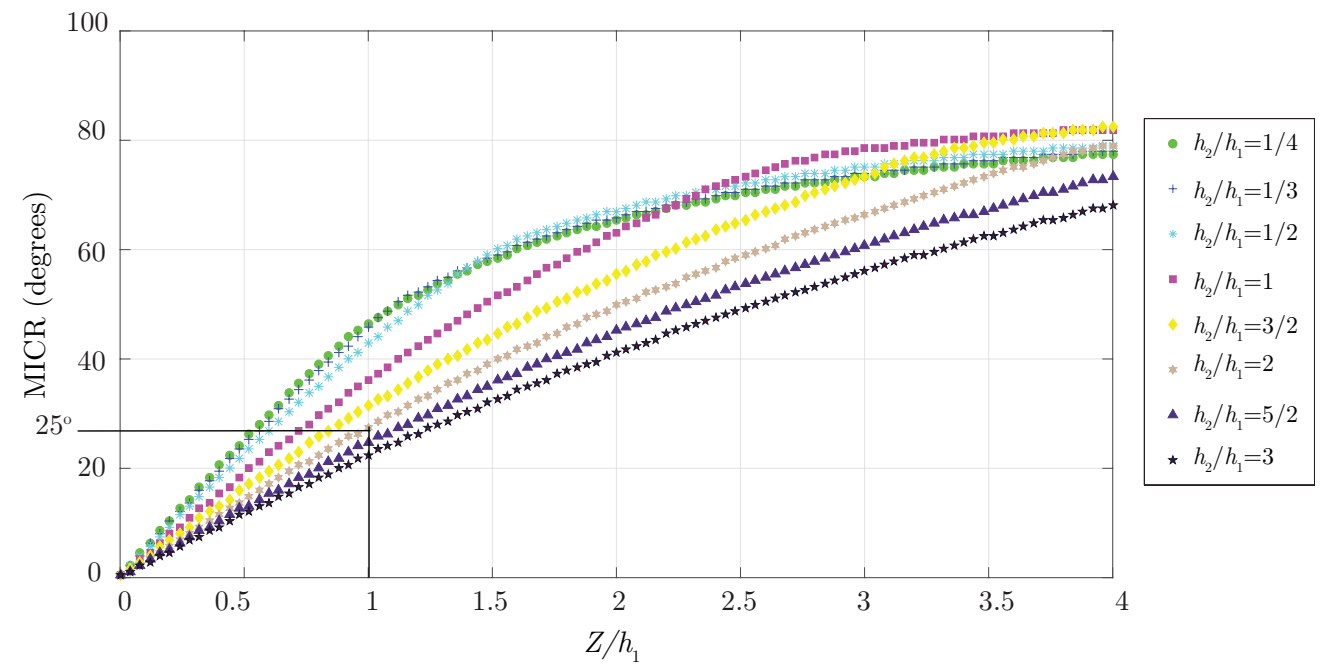

Figure 9: 3-RPS OM1: MICR vs. $\frac{Z}{h_{1}}$ as a function of $\frac{h_{2}}{h_{1}}$ 


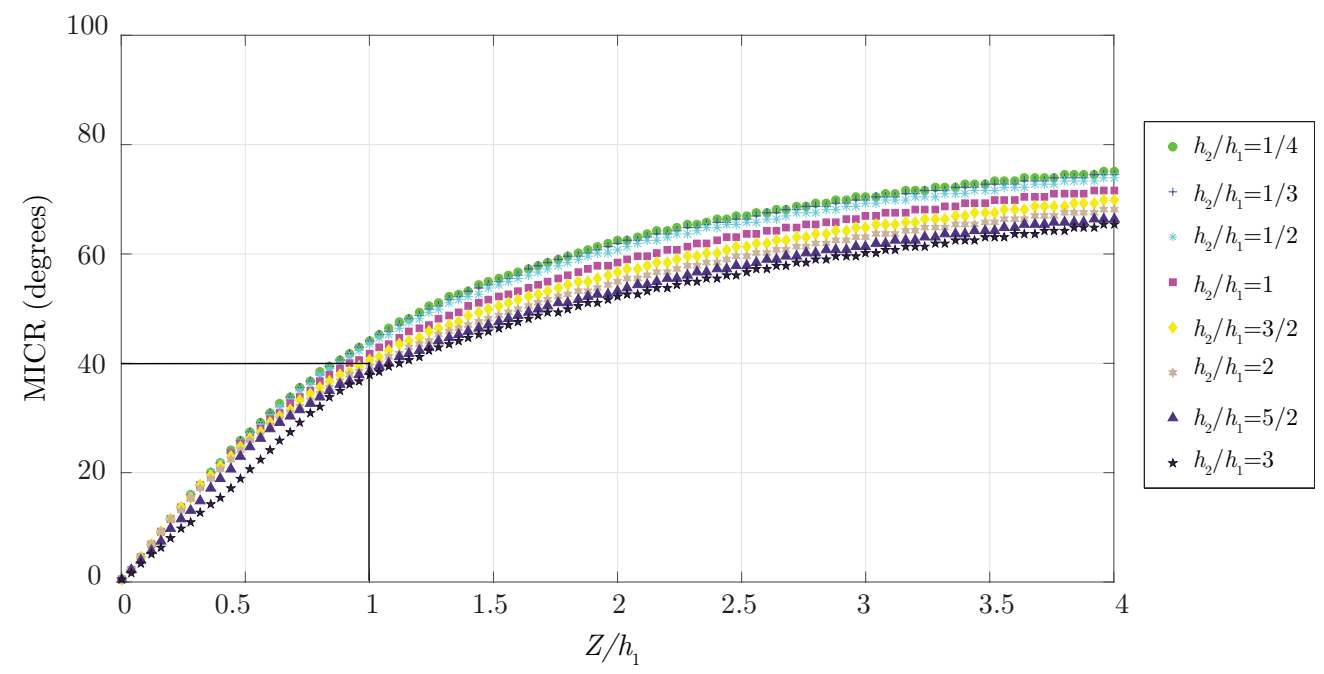

Figure 10: 3-RPS OM2: MICR vs. $\frac{Z}{h_{1}}$ as a function of $\frac{h_{2}}{h_{1}}$

From Eqs. (23) and (24), the maximum displacement within the Maximum Inscribed Circle (MIC) is written as

$$
\mu=\max \left(\sqrt{X^{2}+Y^{2}}\right)=\max \left(h_{2}^{2} \sin \left(\frac{\theta}{2}\right)^{4}\right) \quad \text { when } 0 \leq \theta \leq M I C R ;-\pi \leq \phi \leq \pi
$$

By varying $Z$ from 0 to $4, \theta$ from 0 to MICR and $\phi$ from $-\pi$ to $\pi, \mu$ is plotted for the 3 -RPS PM as shown in Figs. 11 and 12. It is observed that the parasitic motion increases as the ratio $\frac{h_{2}}{h_{1}}$ increases. But, the larger the $\frac{h_{2}}{h_{1}}$ ratio, the smaller the MICR and hence, the smaller the singularity free workspace.

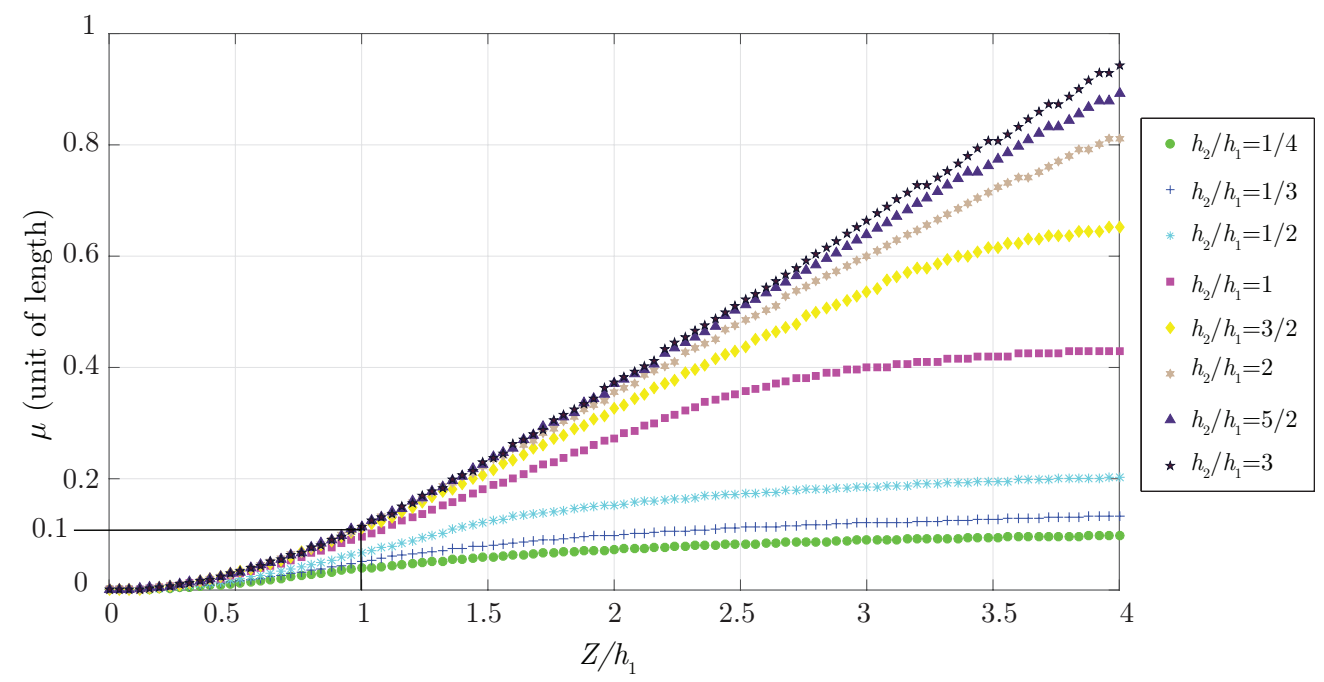

Figure 11: 3-R्PS OM1: $\mu$ vs. $\frac{Z}{h_{1}}$ as a function of $\frac{h_{2}}{h_{1}}$ 


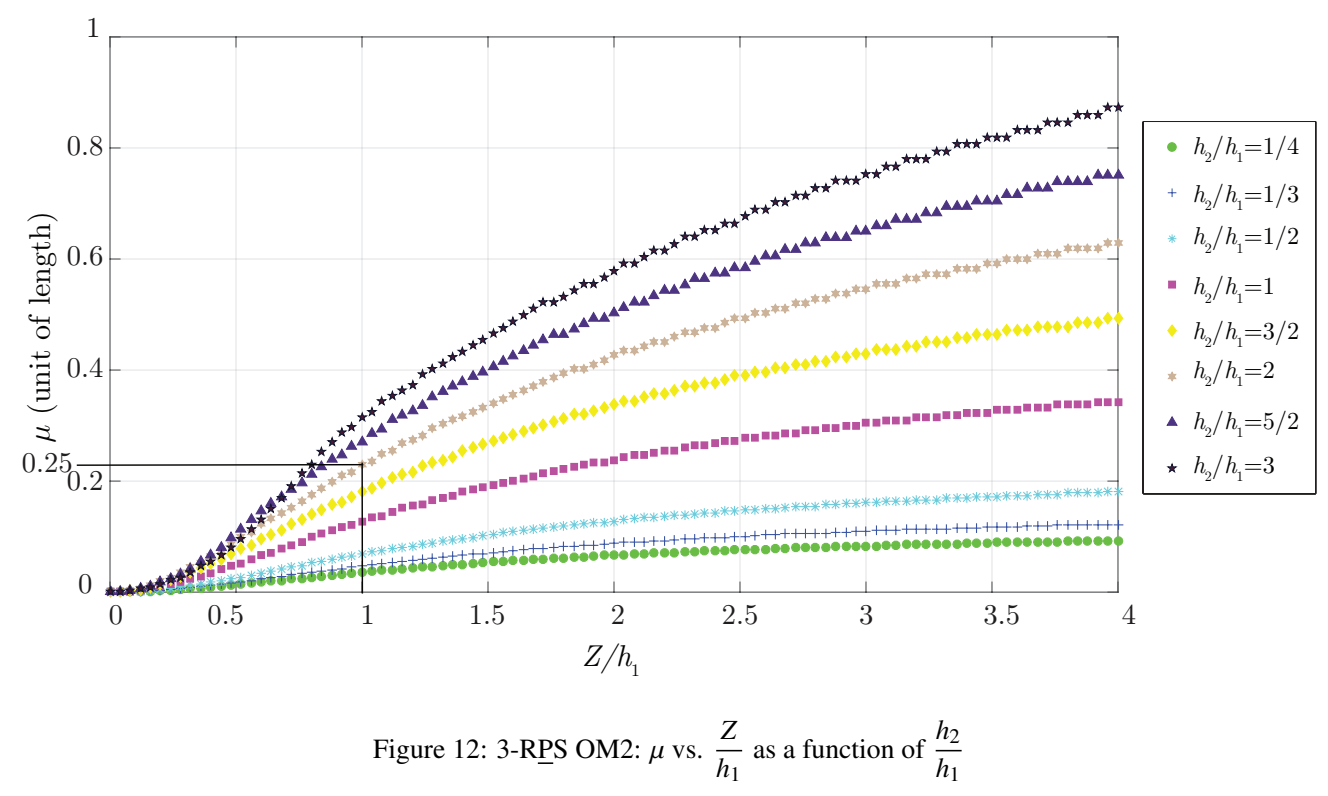

\section{Comparison of 3-[PP]S-Y PMs based on their MICR and parasitic motions}

For most applications, it is better to have a large singularity-free orientation workspace with small parasitic motions. To find out the most suitable manipulator for this purpose, MICR vs. $\mu$ is plotted for all the mechanisms listed in Table 1 in their two operation modes each for three ratios of $\frac{h_{2}}{h_{1}}=\left\{\frac{1}{2}, 1,2\right\}$ and two ratios of $\frac{Z}{h_{1}}=\{1,2\}$. These points are plotted for the abovementioned ratios in Figures 13 to 18. From Eq. (24), it is noticed that parasitic motions are higher for larger singularity free regions. The abscissa is chosen to be $\pi$-MICR since the minimum of this function is preferred and the ordinate is chosen to be $\mu$. For a PM from the 3-[PP]S-Y family, $\pi$-MICR and $\mu$ values are preferred to be smaller. Thus, plotting $\pi$-MICR vs. $\mu$ results in a Pareto front as shown in Figs. 13 to 18 .

Among the manipulators listed in Table 1, it is noticed that the 3-PhPvS PM behaves exactly like the 3-PvPhS $\mathrm{PM}$ and the 3-PhPvS PM like the 3-PvPhS PM. Hence, only the 3-PhPvS PM is considered for analysis. Due to the arrangement of prismatic joints, 3-Pv로 and 3-PhPvS are inherently singular at any configuration and hence they are not considered here. Indeed, for these manipulators, the three actuation forces are coplanar and intersect at a point resulting in only two independent forces. In addition, the PMs 3-RPS and 3-RRS; 3-PhR $\underline{R}$ and 3-PhPvS; behave alike due to identical arrangement of their passive joints. It should be noted that the leg links of the 3-RRS PM are considered to be of length $h_{1}$ so that the moving platform can reach the maximum value of $Z\left(=2 h_{1}\right)$ considered to compare the manipulators at hand.

In Fig. 15, the MICR and $\mu$ values are marked for the 3-RPS PM when $\frac{h_{2}}{h_{1}}=2$ and $\frac{Z}{h_{1}}=1$. These points correspond to the MICR and $\mu$ values spotted in the MICR and $\mu$ vs $\frac{Z}{h_{1}}$ plots in Sec. 6.

To compare the 3-[PP]S-Y family of PMs, their MICR vs $\mu$ values are plotted for different ratios of $\frac{h_{2}}{h_{1}}$ and $\frac{Z}{h_{1}}$ in Figs. 19 and 20. The former figure shows the Pareto optimal solutions listed in Table 2 while the latter shows the solutions that do not dominate any other solutions listed in Table 3 . Their respective configurations are also displayed in these figures. Operation mode 1 is represented with crossed legs or upside down moving platform. But, this need not always be the case. In fact, the generic pose of the 3-[PP]S family of PMs in each of their operation modes is still an open problem. It is noteworthy that the ordinate is chosen to be $\log \mu$ so that the smaller as well as larger values of $\mu$ are clearly visible in the same graph. 


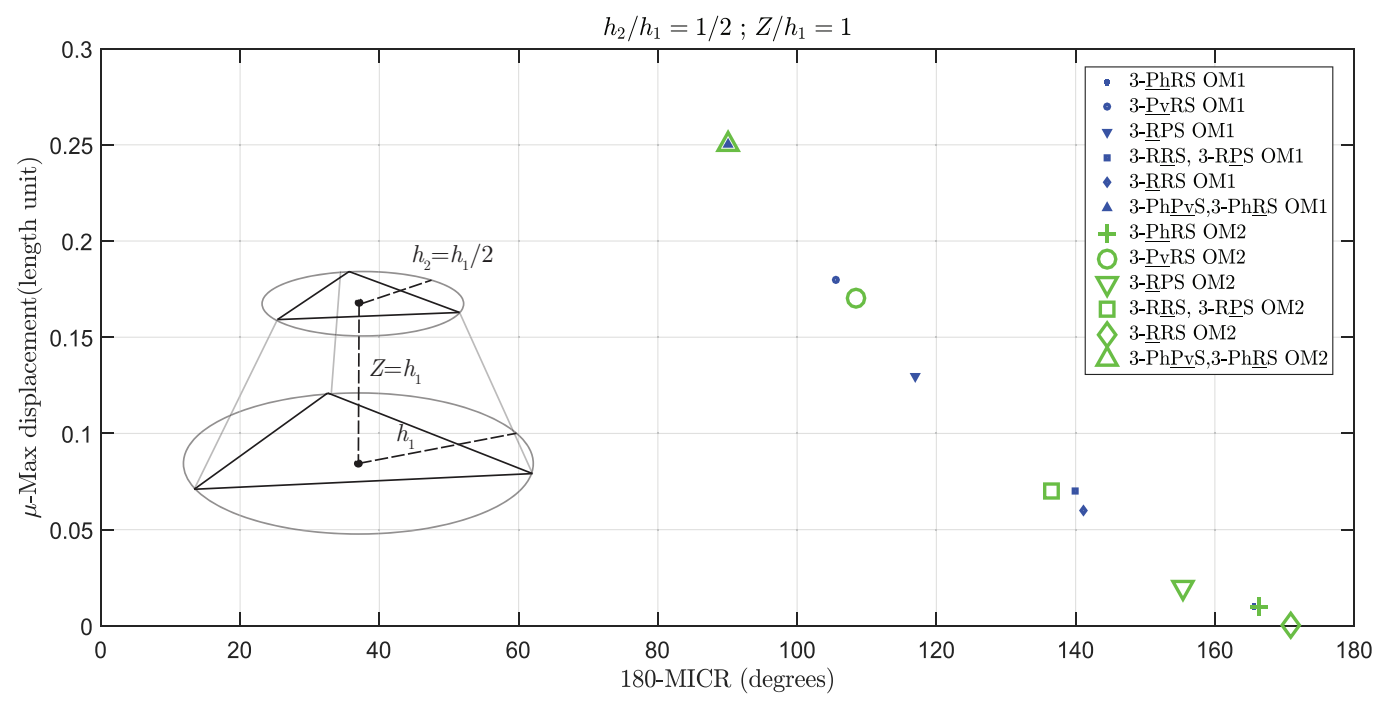

Figure 13: MICR vs maximum displacement $\frac{h_{2}}{h_{1}}=\frac{1}{2}, \frac{Z}{h_{1}}=1$

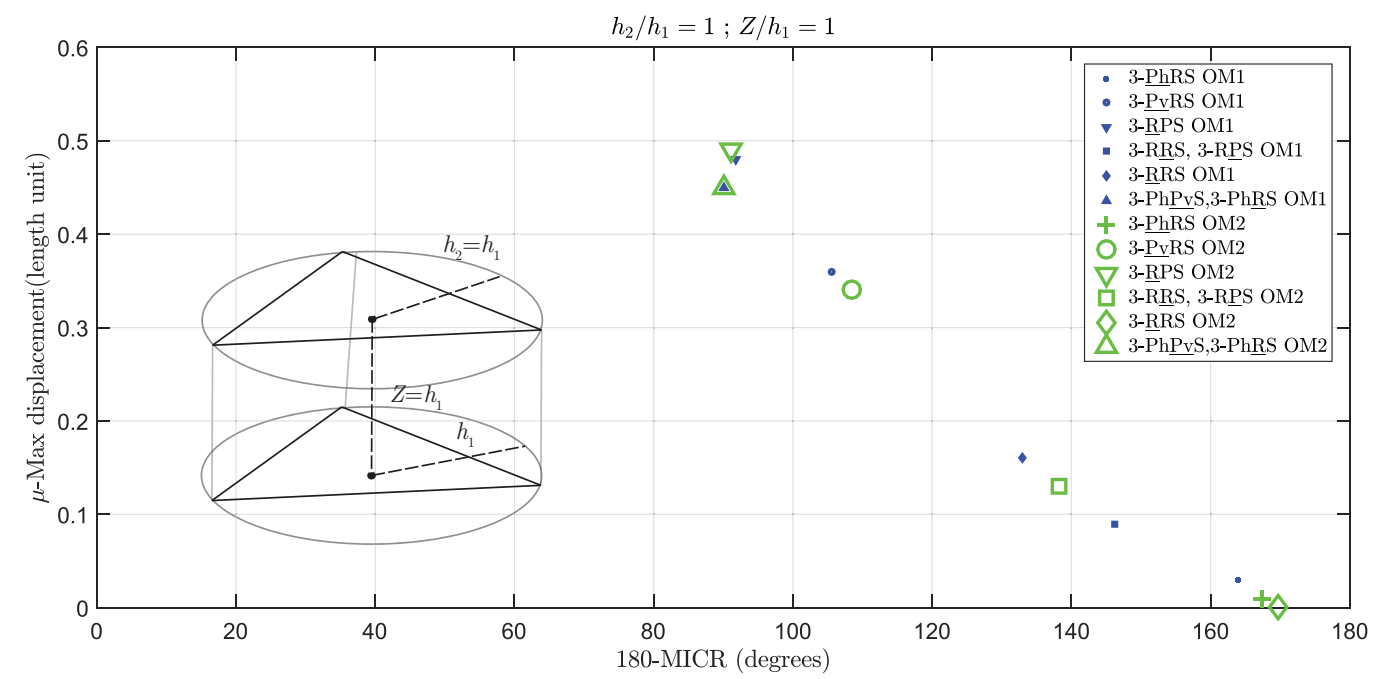

Figure 14: MICR vs maximum displacement $\frac{h_{2}}{h_{1}}=1, \frac{Z}{h_{1}}=1$ 


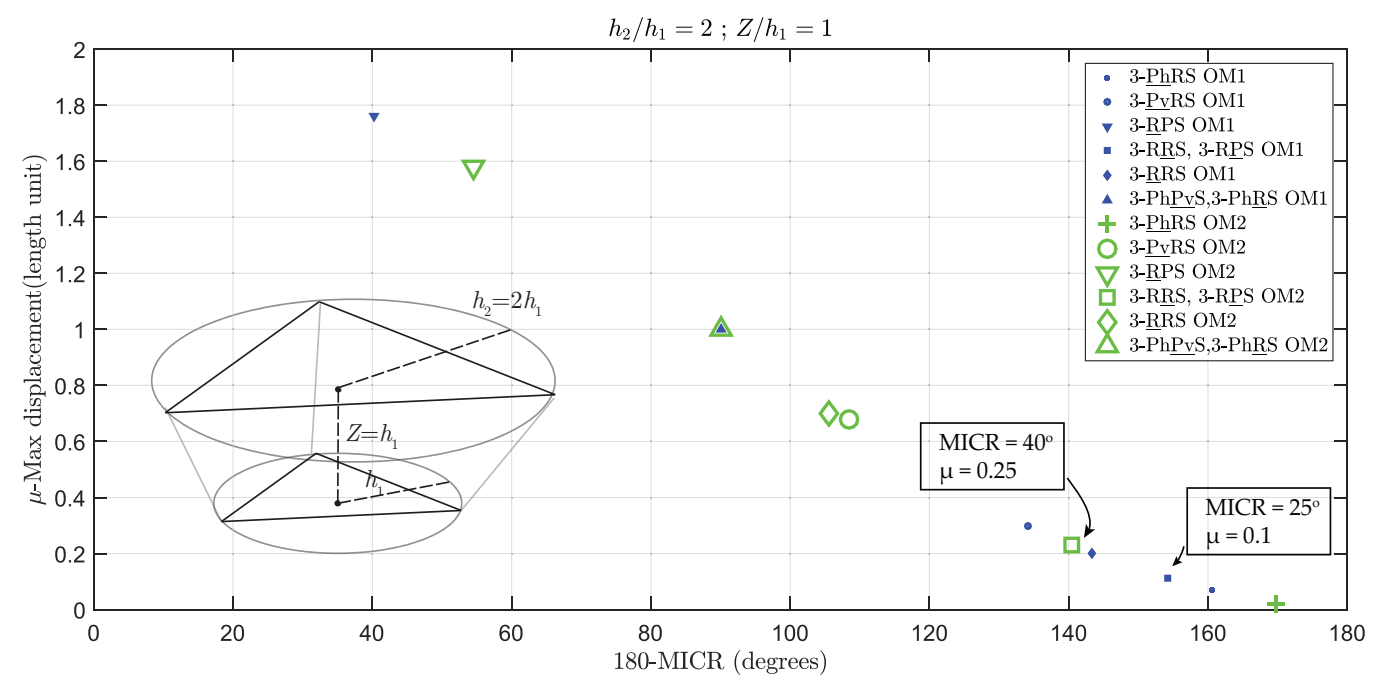

Figure 15: MICR vs maximum displacement $\frac{h_{2}}{h_{1}}=2, \frac{Z}{h_{1}}=1$

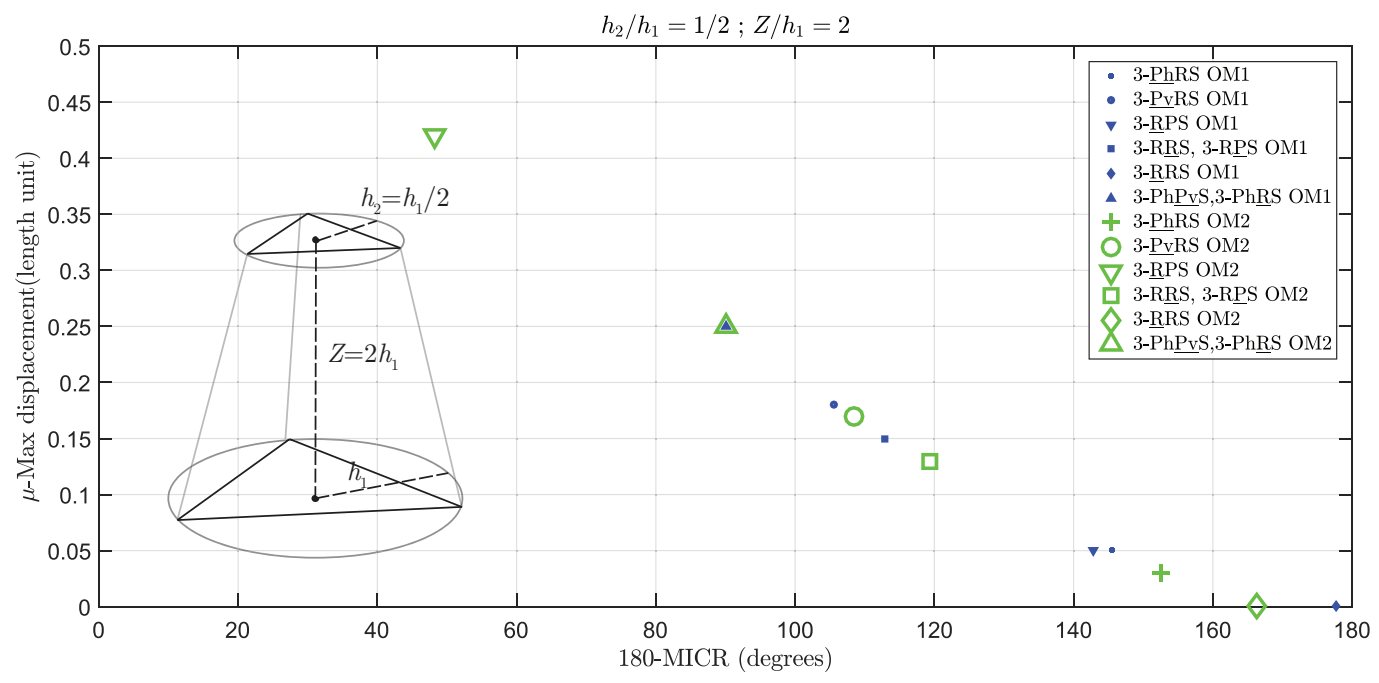

Figure 16: MICR vs maximum displacement $\frac{h_{2}}{h_{1}}=\frac{1}{2}, \frac{Z}{h_{1}}=2$ 


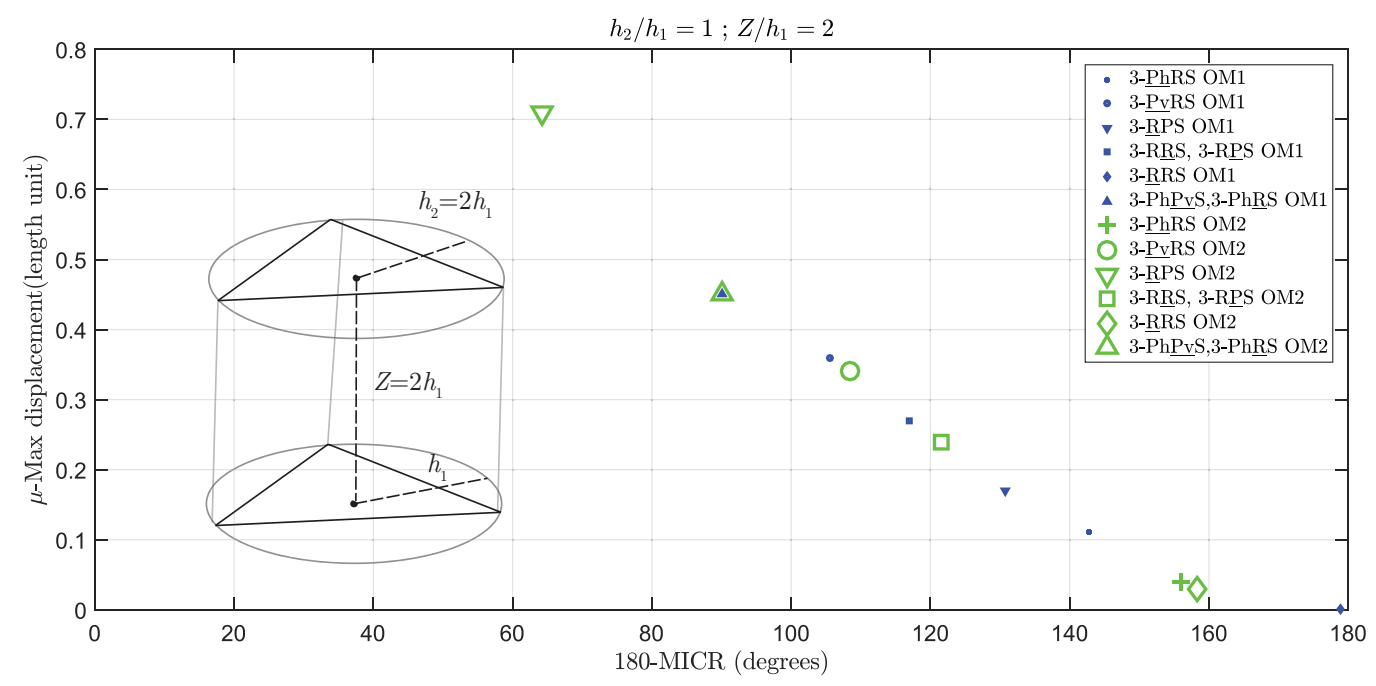

Figure 17: MICR vs maximum displacement $\frac{h_{2}}{h_{1}}=1, \frac{Z}{h_{1}}=2$

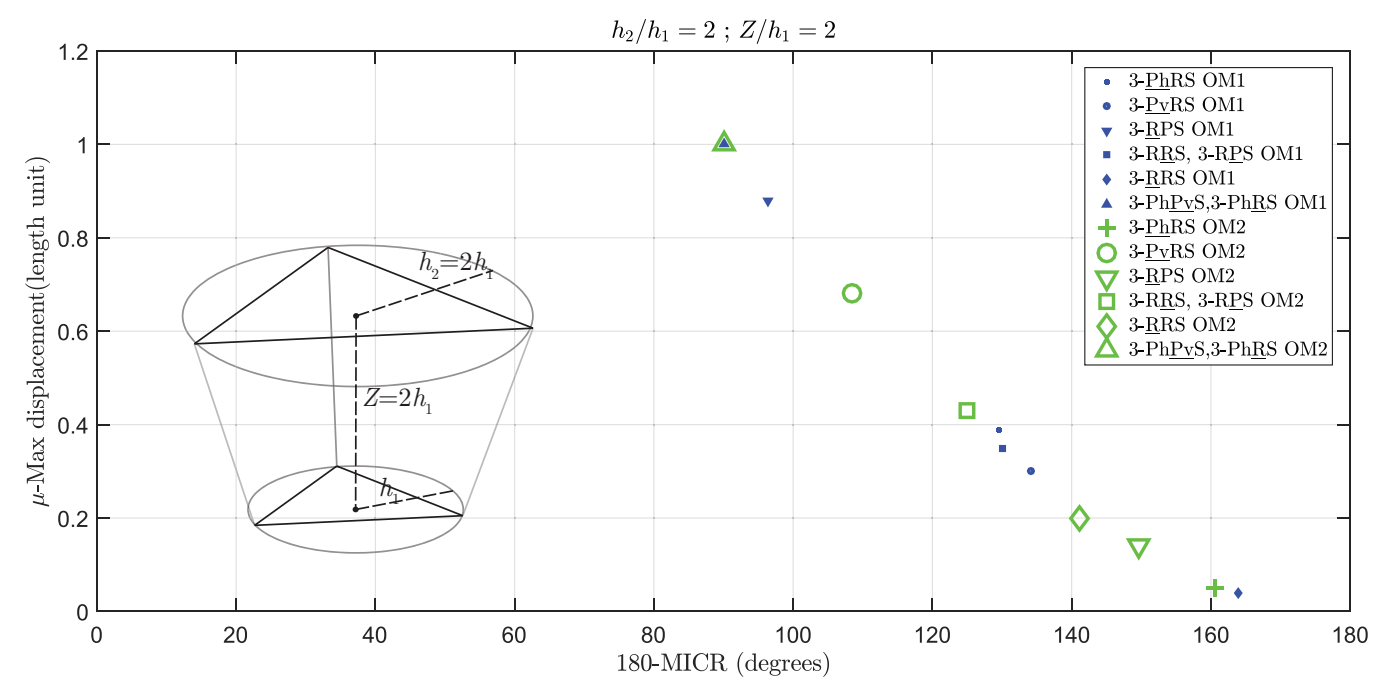

Figure 18: MICR vs maximum displacement $\frac{h_{2}}{h_{1}}=2, \frac{Z}{h_{1}}=2$ 


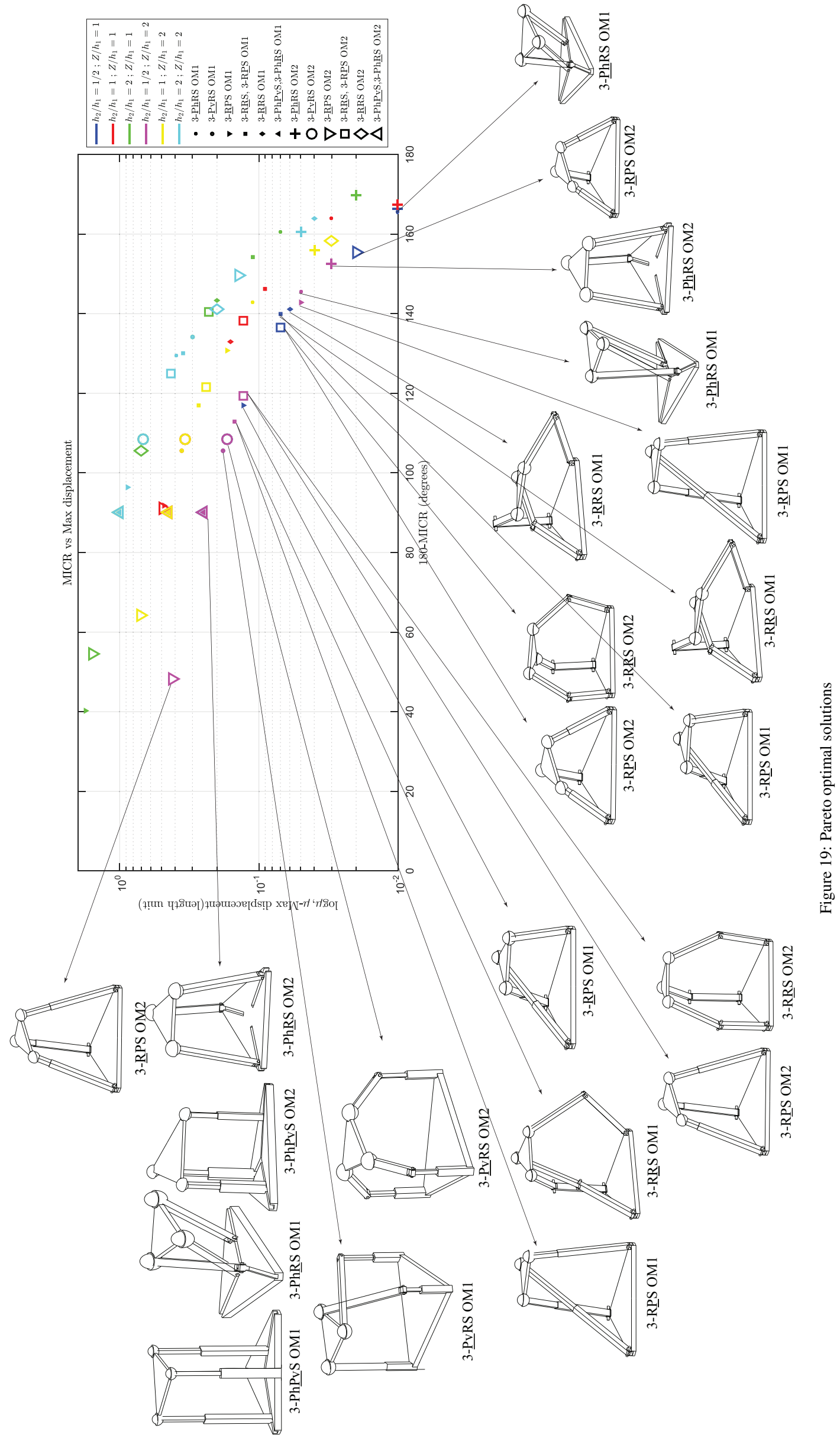




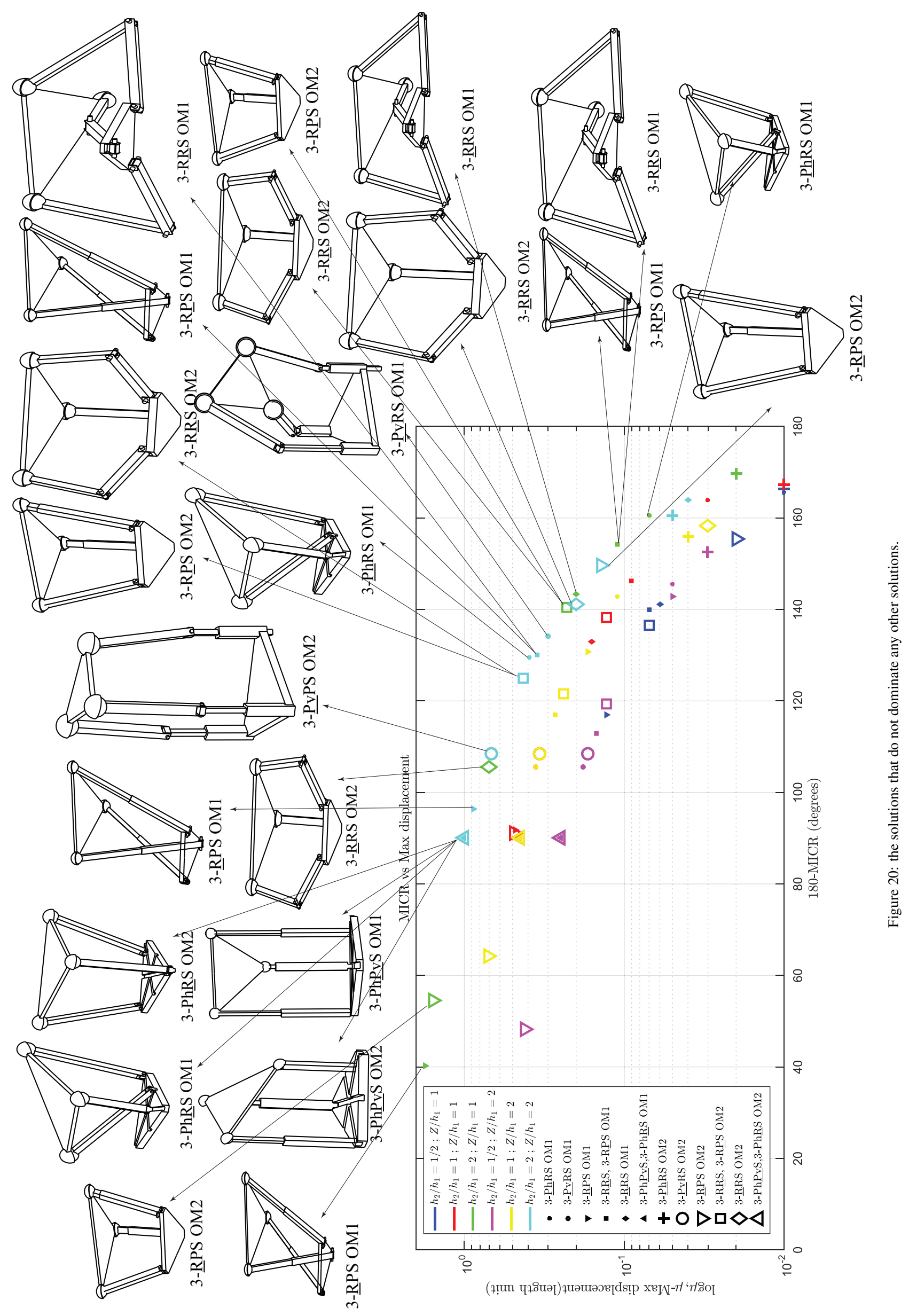




\begin{tabular}{|c|c|c|}
\hline $3-[\mathrm{PP}] \mathrm{S} \mathrm{PM}$ & $\frac{h_{1}}{h_{1}}$ & $\frac{Z}{h_{1}}$ \\
\hline 3-RPS OM2 & $1 / 2$ & \\
\hline 11 - & & \\
\hline 3-Phर्RS OM1 & $1 / 2$ & \\
\hline $3-\mathrm{Ph}$ & $1 / 2$ & \\
\hline $3-\mathrm{P}$ & & \\
\hline $3-\mathrm{Pv}$ & & \\
\hline 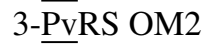 & $1 /$ & \\
\hline 3-RPSS OM1 & $1 / 2$ & \\
\hline & $1 / 2$ & \\
\hline & & \\
\hline $3-\bar{R}]$ & & \\
\hline $3-\mathrm{R} \overline{\mathrm{R}}$ & I & \\
\hline $3-\underline{R} \bar{R} S$ OI & $1 / 2$ & \\
\hline 3-PhRS OM1 & $1 / 2$ & 1,2 \\
\hline 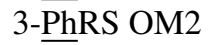 & $1 / 2$ & \\
\hline
\end{tabular}

Table 2: Pareto-optimal solutions (Fig. 19)

\begin{tabular}{|c|c|c|}
\hline 3-[PP]S PM & $\frac{L_{2}}{h_{1}}$ & $\frac{Z}{h_{1}}$ \\
\hline 3-RPS OM1 & & 1,2 \\
\hline $3-\underline{R} P S$ OM2 & & 1,2 \\
\hline 3-PhPvS OM1 & 2 & \\
\hline & & \\
\hline & & \\
\hline $3-\mathrm{Pl}$ & & \\
\hline $3-\underline{R} \bar{R}$ & & 1,2 \\
\hline $3-\overline{\mathrm{Pv}} \mathrm{PS}$ ON & 2 & \\
\hline 3-RPS OM2 & 2 & 1,2 \\
\hline & & 1,2 \\
\hline & & 1,2 \\
\hline & & 1,2 \\
\hline 3-R $\bar{R} S$ & 2 & 1,2 \\
\hline 3-PvRS OM1 & 2 & \\
\hline 3-RRS OM1 & 2 & \\
\hline
\end{tabular}

Table 3: Solutions that do not dominate any other solutions (Fig. 20)

Some observations that can be drawn from these curves are that all the Pareto optimal solutions consist of 3-[PP]SY type PMs with $\frac{h_{2}}{h_{1}}=\frac{1}{2}$ meaning that the circum-radius of the base is double that of the platform. The solutions that do not dominate any other solution include only 3-[PP]S-Y type PMs with $\frac{h_{2}}{h_{1}}=2$ implying that the height of the platform is double the circum-radius of the base. Furthermore, the 3-PhRS PM in operation mode 1 with $\frac{h_{2}}{h_{1}}=\frac{1}{2}$, $\frac{Z}{h_{1}}=1$ and the 3 -PhRS PM in operation mode 2 with $\frac{h_{2}}{h_{1}}=\left\{\frac{1}{2}, 1\right\}, \frac{Z}{h_{1}}=1$ have the least $\mu$ of all the PMs under study. On the contrary, the 3-RPS PM in its first operation mode with $\frac{h_{2}}{h_{1}}=2, \frac{Z}{h_{1}}=1$ has the largest $\mu$.

\section{Ranking the Pareto optimal solutions based on complexity indices}

Some complexity indices were introduced in $[28,27]$ to evaluate the complexity of serial and parallel manipulators at their conceptual design stage. Those indices can be used to rank the parallel manipulators at hand. The complexity is not considered as a separate objective function along with MICR and $\mu$. It is given a lesser priority to compare the PMs belonging to the 3-[PP]S-Y family due to the fact that the complexity indices are independent of $\frac{h_{2}}{h_{1}}$ and $\frac{Z}{h_{1}}$. Moreover, due to little differences between architectures of the 3-[PP]S-Y PMs, most of the indices defined in [27] yield the same complexity. In order to avoid this problem and to rank the Pareto optimal solutions listed in Table 2 in order of complexity, two more indices are introduced in this paper. In total, the following six complexity indices are used to compare the Pareto-optimal solutions listed in Table 2:

\subsection{Joint-number complexity $K_{N}$}

The joint-number complexity $K_{N}$ is defined as

$$
K_{N}=1-\exp \left(-q_{N} N\right)
$$

where $N$ is the number of joints in the PM and $q_{N}$ is the resolution parameter defined by

$$
q_{N}=\left\{\begin{array}{cl}
-\ln (0.1) / N_{\text {max }}, & \text { for } N_{\text {max }}>0 \\
0, & \text { for } N_{\text {max }}=0 .
\end{array}\right.
$$


For all the 3-[PP]S-Y PMs considered here, $N=N_{\max }=9$ and hence $k_{N}=0.9$.

\subsection{Joint-type complexity $K_{J}$}

As the name suggests, the joint-type complexity $K_{J}$ is associated with the type of joints in the PM. The 3-[PP]S-Y PMs consist of only revolute, prismatic and spherical joints and it leads to the definition of $K_{J}$ as follows:

$$
K_{J}=\frac{n_{R} K_{G \mid R}+n_{P} K_{G \mid P}+n_{S} K_{G \mid S}}{n}
$$

where $n_{R}, n_{P}$ and $n_{S}$ are the numbers of revolute, prismatic and spherical joints, respectively with $n=n_{R}+n_{P}+n_{S}$. $K_{G \mid x}$ is the geometric complexity of the pair $x$ as introduced in [27]: $K_{G \mid R}=0.5234, K_{G \mid P}=1$ and $K_{G \mid S}=0$. The values of $K_{J}$ for the Pareto optimal solutions are listed in Table 4.

\subsection{Loop complexity $K_{L}$}

The loop complexity of a PM is defined as:

$$
K_{L}=1-\exp \left(-q_{L} L\right), L=l-l_{m}
$$

where $l$ is the number of kinematic loops and $l_{m}$ is the minimum number of loops required to produce a special displacement group or subgroup. Since each PM belonging to the 3-[PP]S-Y family has non-pure rotations about two horizontal axes and a vertical translation, the three degrees of freedom can be realized using a single kinematic chain. Hence, for all the 3-[PP]S-Y PMs, $l=2$ and $l_{m}=0 . q_{L}$ is defined the same way as in Eq. (31):

$$
q_{L}=\left\{\begin{array}{cl}
-\ln (0.1) / L_{\text {max }}, & \text { for } L_{\max }>0 \\
0, & \text { for } L_{\max }=0 .
\end{array}\right.
$$

where, $L_{\max }=L=2$ for all the Pareto optimal solutions resulting in $K_{L}=0.9$.

\subsection{Link diversity $K_{B}$}

Link diversity $K_{B}$ is defined to quantify the geometric constraints between neighboring joints. For a revolute joint, its axis of rotation is considered whereas for a prismatic joint, its direction. Five possible joint-constraint types between the neighboring joint axes/directions were reported in [27]:

1. Type $B_{1}$ : Orthogonal intersection.

2. Type $B_{2}$ : Nonorthogonal intersection.

3. Type $B_{3}$ : Parallelism.

4. Type $B_{4}$ : Orthogonal but not intersecting.

5. Type $B_{5}$ : Skew.

Thus, the geometric-constraint diversity was defined as:

$$
K_{B}=\frac{B}{B_{\max }}, \quad B=-\sum_{i=1}^{c} b_{i} \log _{2}\left(b_{i}\right), \quad b_{i}=\frac{M_{i}}{\sum_{i=1}^{c} M_{i}},
$$

where $B$ is the entropy of the joint-constraint types and $B_{\max }=2.32$ [27]. $c$ is the number of distinct joint-constraint types and $M_{i}$ is the number of instances of each type of joint-constraints.

For 3-[PP]S-Y PMs, the joint-constraint between the first two joints in each limb is of type $B_{1}$ except for the 3-RRS PM where the revolute joint axes are parallel and is of type $B_{3}$. The constraint type is always $B_{5}$ between the second and the spherical joint while it is either $B_{2}$ or $B_{3}$ between different limbs. There are three instances of each type due to three limbs and hence for any 3 -[PP]S-Y PM, $b_{i}=\frac{3}{9}, i=1,2,3$. This leads to $B=\log _{2}(3)$ and $K_{B}=0.6832$.

Two more complexity indices are introduced in this paper to facilitate the ranking of the Pareto optimal 3-[PP]S-Y PMs: 


\subsection{Actuator-position complexity $K_{P}$}

Actuator-position complexity $K_{P}$ is defined as:

$$
\frac{1}{m} \sum_{i=1}^{m} \frac{p_{i}-1}{n_{i}},
$$

where $m$ is the number of limbs, $n_{i}$ is the total number of joints in the $i$-th limb and $p_{i}$ is the location of the actuated joint i.e. $p_{i}=k$ if $k$-th joint is actuated. For 3-[PP]S-Y PMs, $K_{P}=0$ if the first joint is actuated and $K_{P}=1 / 3$ if the second joint is actuated. It is noteworthy that the actuator position index can be applied to any other serial kinematic chain or a PM even if the position of the actuator differs from one limb to another.

\subsection{Operation mode complexity $K_{O M}$}

Operation mode complexity $K_{O M}$ is defined specifically for 3-[PP]S-Y PMs. As seen in Sec. 4, a 3-[PP]S-Y PM has two different operation modes. In the first operation mode, the moving platform is rotated upside down by 180 degrees with respect to the fixed base. As a result of this complexity analysis, it is prone to have internal collisions as compared to operation mode 2, as shown for a 3-RPS PM in Sec. 6. Although a detailed collision analysis is necessary to overcome this issue, it is partially overcome in this paper by introducing a new complexity index as follows:

$$
\begin{aligned}
& K_{O M}=1 \text { for OM1 } \\
& K_{O M}=0 \text { for OM2 }
\end{aligned}
$$

\subsection{Total complexity}

Total complexity $K \in[0,1]$ of a kinematic chain was defined as a convex combination of the different complexity indices [27]. In this context, for the 3-[PP]S-Y PMs, it is defined as

$$
K=w_{N} K_{N}+w_{J} K_{J}+w_{L} K_{L}+w_{B} K_{B}+w_{P} K_{P}+w_{O M} K_{O M}
$$

where $w_{N}, w_{J}, w_{L}, w_{B}, w_{P}$ and $w_{O M}$ denote their corresponding weights, such that

$$
w_{N}+w_{J}+w_{L}+w_{B}+w_{P}+w_{O M}=1
$$

Assigning equal weights ${ }^{5}$ to all complexity indices implies

$$
K=\frac{1}{6}\left(K_{N}+K_{J}+K_{L}+K_{B}+K_{P}+K_{O M}\right)
$$

The Pareto optimal solutions listed in Table 2 are ranked in ascending order of complexity in Table 4. It is apparent that the 3-RPS, 3-PvRS and 3-PhRS PMs in OM2 dominate their counterparts with higher singularity-free orientation workspaces, lower parasitic motions and are the simplest ones, their complexity being equal to 0.4985 . The 3-PhPvS $\mathrm{PM}$ in $\mathrm{OM} 1$ is the most complex Pareto optimal solution with a complexity equal to 0.7472 .

\section{Conclusion}

In this paper, different operation and actuation modes of the 3-[PP]S-Y zero torsion parallel manipulators were compared based on their singularity free orientation workspace and parasitic motions. The manipulators belonging to the 3-[PP]S-Y family of PMs were considered and kinematic constraint equations were derived for their general architectures. A primary decomposition of the ideal of the constraint polynomials showed that they have two operation modes. Furthermore, their forward kinematic Jacobian matrices were derived for the mechanisms at hand. The

\footnotetext{
${ }^{5}$ Since $K_{N}, K_{L}$ and $K_{B}$ are the same for all the 3-[PP]S-Y PMs, the weights $w_{N}, w_{L}$ and $w_{B}$ do not affect the complexity ranking. However, when $w_{L}=\frac{1}{3}$ and $w_{P}=\frac{1}{12}, 3$-RRS PM in OM2 becomes the simplest PM belonging to the 3-[PP]S-Y family. Eventually, the weights can be chosen according to the application.
} 


\begin{tabular}{|c|c|c|c|c|c|c|c|c|}
\hline Rank & Pareto optimal solutions & $K_{N}$ & $K_{J}$ & $K_{L}$ & $K_{B}$ & $K_{P}$ & $K_{O M}$ & $K$ \\
\hline \multirow[t]{3}{*}{1} & 3-RPS OM2 & 0.9 & 0.5078 & 0.9 & 0.6832 & 0 & 0 & 0.4985 \\
\hline & 3-PvRS OM2 & 0.9 & 0.5078 & 0.9 & 0.6832 & 0 & 0 & 0.4985 \\
\hline & $3-\overline{\mathrm{Ph}} \mathrm{RS}$ OM2 & 0.9 & 0.5078 & 0.9 & 0.6832 & 0 & 0 & 0.4985 \\
\hline 2 & 3-RRS OM2 & 0.9 & 0.3489 & 0.9 & 0.6832 & 0.3333 & 0 & 0.5276 \\
\hline \multirow[t]{2}{*}{3} & 3-PhRS OM2 & 0.9 & 0.5078 & 0.9 & 0.6832 & 0.3333 & 0 & 0.5541 \\
\hline & 3-R्PS OM2 & 0.9 & 0.5078 & 0.9 & 0.6832 & 0.3333 & 0 & 0.5541 \\
\hline 4 & 3-PhPvS OM2 & 0.9 & 0.6667 & 0.9 & 0.6832 & 0.3333 & 0 & 0.5805 \\
\hline 5 & 3-ㅁRS OM1 & 0.9 & 0.3489 & 0.9 & 0.6832 & 0 & 1 & 0.6387 \\
\hline \multirow[t]{2}{*}{6} & 3-PvRS OM1 & 0.9 & 0.5078 & 0.9 & 0.6832 & 0 & 1 & 0.6552 \\
\hline & $3-\overline{\mathrm{Ph}} \mathrm{RS}$ OM1 & 0.9 & 0.5078 & 0.9 & 0.6832 & 0 & 1 & 0.6552 \\
\hline 7 & 3-RRS OM1 & 0.9 & 0.3489 & 0.9 & 0.6832 & 0.3333 & 1 & 0.6942 \\
\hline \multirow[t]{2}{*}{8} & 3-PhRS OM1 & 0.9 & 0.5078 & 0.9 & 0.6832 & 0.3333 & 1 & 0.7207 \\
\hline & 3-R्PS OM1 & 0.9 & 0.5078 & 0.9 & 0.6832 & 0.3333 & 1 & 0.7207 \\
\hline 9 & 3-Ph $\underline{\mathrm{P} v S}$ OM1 & 0.9 & 0.6667 & 0.9 & 0.6832 & 0.3333 & 1 & 0.7472 \\
\hline
\end{tabular}

Table 4: Complexity indices of Pareto optimal 3-[PP]S-Y PMs in ascending order

determinant of the Jacobian matrix was expressed as a function of the orientation workspace parameters tilt, azimuth and height of the moving-platform, $Z$. The vanishing condition of the determinant was used to visualize the singularity loci in the orientation workspace. An index, named Maximum Inscribed Circle Radius (MICR), was used to quantify the singularity free orientation workspace of the manipulators. The maximum parasitic motion within the Maximum Inscribed Circle denoted as $\mu$ and was used as a second performance index. For $\frac{h_{2}}{h_{1}}=\frac{1}{2}, 1,2$ and $\frac{Z}{h_{1}}=1,2$, MICR vs $\mu$ values were plotted for all the mechanisms in Table 1. The 3-RPS PM was taken as an example to illustrate the methodology to find the MICR and $\mu$ values. For all manipulators belonging to the 3-[PP]S-Y family, the plots of (180-MICR) vs. $\mu$ were drawn such that a minimum of these objective functions is the preferred solution. Different plots were drawn for different ratios of the circumference of the moving-platform to the circumference of the fixed base. Ultimately, $\left(180\right.$-MICR) vs. $\log _{10}(\mu)$ points are plotted in the same graph for all the mechanisms for easier comparison. A Pareto front was observed in the plot and manipulator configurations were represented for the Paretooptimal solutions and the solutions that do not dominate any other solution. It was concluded that the Pareto optimal solutions only include the PMs from the 3-[PP]S-Y family with the circum-radius of the base twice as that of the platform. The solutions that do not dominate any other solutions include the PMs from the 3-[PP]S-Y family with the circum-radius of the platform twice as that of the base. Therefore, the MICR vs $\mu$ plots could be used to compare the 3-[PP]S family of PMs especially while choosing them for a particular application. Furthermore, the complexity indices of the Pareto optimal solutions were calculated. For equal weights of all complexity indices, it was shown that the 3-RPS, 3-PvRS and 3-PhRS PMs in their second operation mode are the simplest ones amongst 3-[PP]S-Y PMs. Future work will include a comparison between 3-[PP]S- $\Delta$ and 3-[PP]S-cube PMs for different actuation schemes. Furthermore, the comparison will be done while taking into account joint limits and self collisions. Additionally, MICR, parasitic motions and proposed complexity indices can be used as a basis of comparison for other lower mobility PMs. 


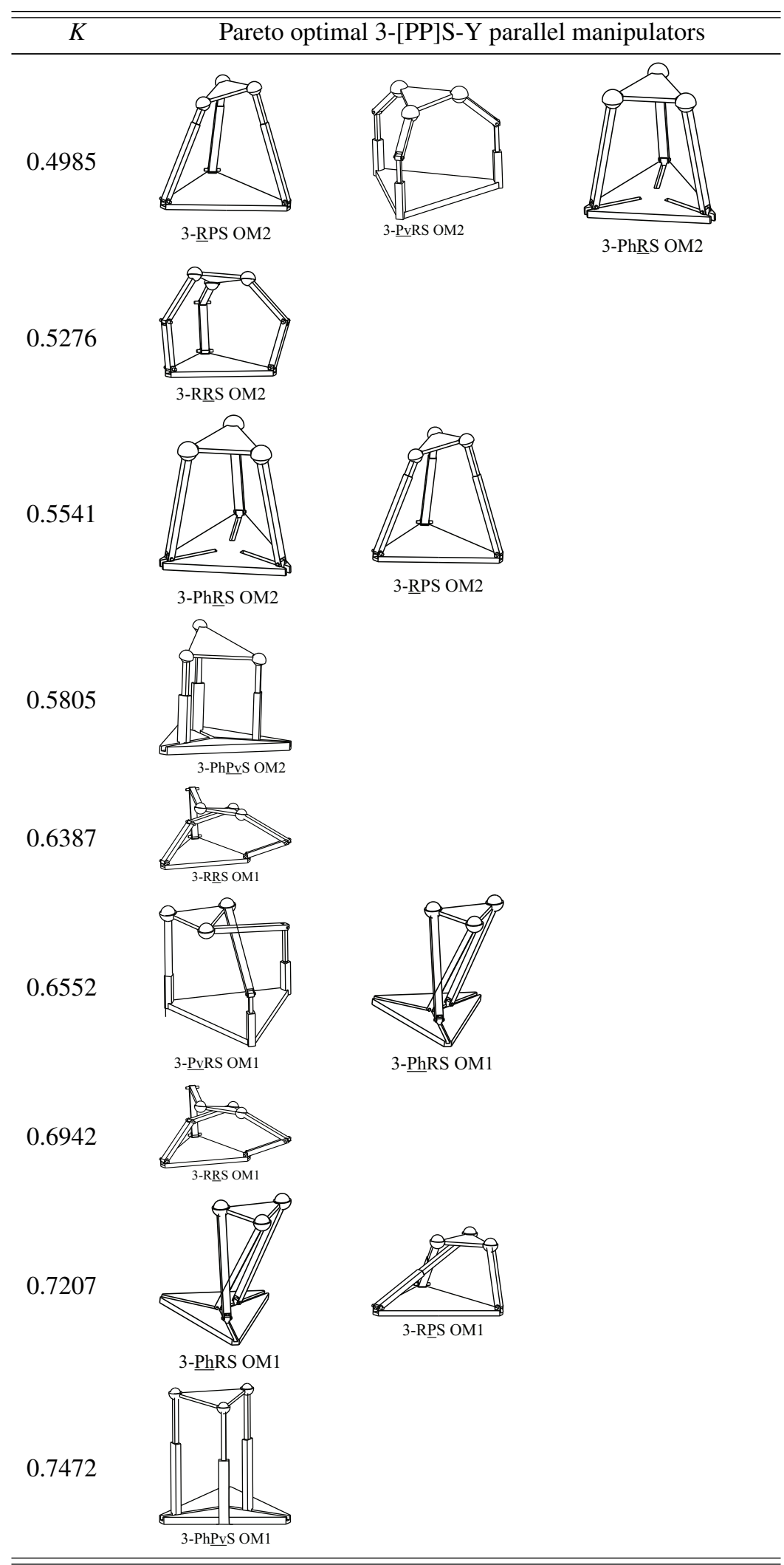

Table 5: Pareto optimal 3-[PP]S-Y parallel manipulators configurations in ascending order of complexity 


\section{Acknowledgments}

This work was conducted with the support of both Centrale Nantes and the French National Research Agency (ANR project Kapamat \#ANR-14-CE34-0008-01).

\section{References}

[1] J. P. Merlet, Parallel Robots, 2nd Edition, Springer Publishing Company, Incorporated, 2010.

[2] K. Hunt, Structural kinematics of in-parallel-actuated robot-arms, ASME J. Mech., Transm., Autom. Des 105 (4) (1983) 705-712.

[3] G. Wu, S. Bai, P. Hjornet, Architecture optimization of a parallel schoenflies-motion robot for pick-and-place applications in a predefined workspace, Mechanism and Machine Theory 106 (2) (2016) 148-165.

[4] G. Wu, S. Bai, S. Caro, Transmission quality evaluation for a class of four-limb parallel schoenflies-motion generators with articulated platforms, in: In : Zeghloul S., Romdhane L., Laribi M. (eds) Computational Kinematics. Mechanisms and Machine Science, Springer, Cham., Vol. 50, 2018, pp. 282-290.

[5] Q. C. Li, Z. Huang, A family of symmetrical lower-mobility parallel mechanisms with spherical and parallel subchains, Journal of Robotic Systems 20 (6) (2003) 297-305.

[6] R. Clavel, Delta, a fast robot with parallel geometry, in: C. W. Burckhardt (Ed.), Proc of the 18th International Symposium on Industrial Robots, Springer-Verlag, New York, 1988, pp. 91-100.

[7] L.-W. Tsai, Kinematics of a three-dof platform with three extensible limbs, in: J. Lenarčič, V. Parenti-Castelli (Eds.), Recent Advances in Robot Kinematics, Springer Netherlands, Dordrecht, 1996, pp. 401-410.

[8] C. Gosselin, J. Angeles, The optimum kinematic design of a spherical three-degree-of-freedom parallel manipulator, Journal of Mechanisms, Transmissions, and Automation in Design 111 (2) (1989) 202-207.

[9] I.A.Bonev, D.Zlatanov, C.M.Gosselin, Advantages of the modified euler angles in the design and control of PKMs, in: 2002 Parallel Kinematic Machines International Conference, 2002, pp. 171-188.

[10] I. A. Bonev, Direct kinematics of zero-torsion parallel mechanisms, in: 2008 IEEE International Conference on Robotics and Automation, 2008, pp. 3851-3856.

[11] X.-J. Liu, I. A. Bonev, Orientation capability, error analysis, and dimensional optimization of two articulated tool heads with parallel kinematics, Journal of Manufacturing Science and Engineering 130 (1) (2008) 011015-011015-9.

[12] K. M. Lee, D. K. Shah, Kinematic analysis of a three-degrees-of-freedom in-parallel actuated manipulator, IEEE Journal on Robotics and Automation 4 (3) (1988) 354-360.

[13] K. J. Waldron, M. Raghavan, B. Roth, Kinematics of a hybrid series-parallel manipulation system, Journal of Dynamic Systems, Measurement, and Control 111 (2) (1989) 211-221.

[14] S.-M. Song, M.-D. Zhang, A study of reactional force compensation based on three-degree-of freedom parallel platforms, Journal of Robotic Systems 12 (12) (1995) 783-794.

[15] J. A. Carretero, R. P. Podhorodeski, M. A. Nahon, C. M. Gosselin, Kinematic analysis and optimization of a new three degree-of-freedom spatial parallel manipulator, Vol. 122, ASME, 1999, pp. 17-24.

[16] J. Wahl, Articulated tool head, US Patent 6,431,802 (Aug. 13 2002).

[17] J. D. López, C. F. Rodríguez, Design of a boat simulator using two parallel manipulators, in: Proceedings of ASME 2014 International Mechanical Engineering Congress and Exposition IMECE, November 14-20, 2014, Montreal, Canada.

[18] H. Tetik, R. Kalla, G. Kiper, S. Bandyopadhyay, Position kinematics of a 3-RRS parallel manipulator, in: V. Parenti-Castelli, W. Schiehlen (Eds.), ROMANSY 21 - Robot Design, Dynamics and Control: Proceedings of the 21st CISM-IFToMM Symposium, June 20-23, Udine, Italy, Springer International Publishing, Cham, 2016, pp. 65-72.

[19] G. Yang, T. J. Teo, I. M. Chen, W. Lin, Analysis and design of a 3-dof flexure-based zero-torsion parallel manipulator for nano-alignment applications, in: 2011 IEEE International Conference on Robotics and Automation, 2011, pp. 2751-2756.

[20] L. Nurahmi, J. Schadlbauer, S. Caro, M. Husty, P. Wenger, Kinematic analysis of the 3-RPS cube parallel manipulator, Journal of Mechanisms and Robotics 7 (1) (2015) 011008-011008-11.

[21] L. W. Tsai, S. Joshi, Comparison study of architectures of four 3 degree-of-freedom translational parallel manipulators, in: Proceedings 2001 ICRA. IEEE International Conference on Robotics and Automation (Cat. No.01CH37164), Vol. 2, 2001, pp. 1283-1288 vol.2.

[22] G. Pond, J. A. Carretero, Quantitative dexterous workspace comparison of parallel manipulators, Mechanism and Machine Theory 42 (10) (2007) $1388-1400$

[23] Q. Li, Z. Chen, Q. Chen, C. Wu, X. Hu, Parasitic motion comparison of 3-PRS parallel mechanism with different limb arrangements, Robotics and Computer-Integrated Manufacturing 27 (2) (2011) 389-396.

[24] Q. Li, J. M. Hervé, 1T2R parallel mechanisms without parasitic motion, IEEE Transactions on Robotics 26 (3) (2010) 401-410.

[25] A. Nayak, L. Nurahmi, P. Wenger, S. Caro, Comparison of 3-RPS and 3-SPR parallel manipulators based on their maximum inscribed singularity-free circle, in: P. Wenger, P. Flores (Eds.), New Trends in Mechanism and Machine Science: Theory and Industrial Applications, Springer International Publishing, Cham, 2017, pp. 121-130.

[26] J. Schadlbauer, D. R. Walter, M. L. Husty, A complete kinematic analysis of the 3-RPS parallel manipulator, in: 15th National Conference on Machines and Mechanisms (NaCoMM 2011), Chennai, India, Nov, 2011.

[27] W. A. Khan, S. Caro, J. Angeles, D. Pasini, A Formulation of Complexity-Based Rules for the Preliminary Design Stage of Robotic Architectures, International Conference on Engineering Design, ICED07, August 28-31, Paris, France, 2007.

[28] S. Caro, W. A. Khan, D. Pasini, J. Angeles, The rule-based conceptual design of the architecture of serial schnflies-motion generators, Mechanism and Machine Theory 45 (2) (2010) $251-260$. 
[29] L. Nurahmi, S. Caro, P. Wenger, Operation modes and singularities of 3-PRS parallel manipulators with different arrangements of P-joints, in: ASME 2015 International Design Engineering Technical Conferences and Computers and Information in Engineering Conference, American Society of Mechanical Engineers, 2015, pp. V05CT08A015-V05CT08A015.

[30] J. Schadlbauer, L. Nurahmi, M. Husty, P. Wenger, S. Caro, Operation modes in lower-mobility parallel manipulators, in: A. Kecskeméthy, F. Geu Flores (Eds.), Interdisciplinary Applications of Kinematics: Proceedings of the International Conference, Lima, Peru, September 9-11, 2013, Springer International Publishing, Cham, 2015, pp. 1-9.

[31] S. Maraje, L. Nurahmi, S. Caro, Operation modes comparison of a reconfigurable 3-PRS parallel manipulator based on kinematic performance, in: ASME 2016 International Design Engineering Technical Conferences and Computers and Information in Engineering Conference, American Society of Mechanical Engineers, 2016, pp. V05BT07A055-V05BT07A055.

[32] D. Zlatanov, I. A. Bonev, C. M. Gosselin, Constraint singularities as c-space singularities, in: J. Lenarčič, F. Thomas (Eds.), Advances in Robot Kinematics: Theory and Applications, Springer Netherlands, Dordrecht, 2002, pp. 183-192.

[33] M. L. Husty, M. Pfurner, H.-P. Schröcker, K. Brunnthaler, Algebraic methods in mechanism analysis and synthesis, Robotica 25 (6) (2007) $661-675$.

[34] M. L. Husty, H.-P. Schröcker, Algebraic geometry and kinematics, in: I. Z. Emiris, F. Sottile, T. Theobald (Eds.), Nonlinear Computational Geometry, Springer New York, NY, 2010, pp. 85-107.

[35] S. A. Joshi, L.-W. Tsai, Jacobian analysis of limited-dof parallel manipulators, Journal of Mechanical Design 124 (2) (2002) $254-258$.

[36] I. A. Bonev, J. Ryu, Orientation workspace analysis of 6-dof parallel manipulators, in: Proceedings of the ASME, 1999. 